\title{
Circuit Design Steps for Nano-Crossbar Arrays: Area-Delay-Power Optimization with Fault Tolerance
}

\author{
M. Ceylan Morgul*, Luca Frontini, Onur Tunali, Lorena Anghel, Valentina Ciriani, E. Ioana Vatajelu, Csaba \\ Andras Moritz, Mircea R. Stan, Dan Alexandrescu, and Mustafa Altun
}

\begin{abstract}
Nano-crossbar arrays have emerged to achieve high performance computing beyond the limits of current CMOS with the drawback of higher fault rates. They offer area and power efficiency in terms of their easy-to-fabricate and dense physical structures. They consist of regularly placed crosspoints as computing elements, which behave as diode, memristor, field effect transistor, or novel four-terminal switching devices. In this study, we establish a complete design framework for crossbar circuits explaining and analyzing every step of the process. We comparatively elaborate on these technologies in the sense of their capabilities for computation regarding area including a new logic synthesis technique for memristors, fault tolerance including a novel paradigm for four-terminal devices, delay, and power consumption. As a result, this study introduces a synthesis methodology that considers basic technology preference for switching crosspoints and fault rates of the given crossbar as well as their effects on performance metrics including power, delay, and area.
\end{abstract}

Index Terms-Crossbar Arrays, Logic Synthesis, Defect Tolerance, Fault Tolerance, Performance Optimization, Memristor Arrays

\section{INTRODUCTION}

Nano-crossbars arrays have emerged to be an alternative/complementary technology to CMOS [46]. In their fabrication, relatively cheap bottom-up nano-fabrication techniques are used rather than pure lithography based conventional

M. Ceylan Morgul and Mustafa Altun are with the Department of Electronics and Communication Engineering, and Onur Tunali is with the Department of Nanoscience and Nanoengineering of Istanbul Technical University, Istanbul, Turkey e-mail: \{morgul, onur.tunali, altunmus\}@itu.edu.tr

Luca Frontini and Valentina Ciriani are with the Dipartimento di Informatica, Università degli Studi di Milano, Milan, Italy e-mail: \{luca.frontini, valentina.ciriani\}@unimi.it

Lorena Anghel and E. Ioana Vatajelu are with TIMA laboratory, Grenoble-Alpes University, Grenoble, France e-mail: \{lorena.anghel, ioana.vatajelu\}@univ-grenoble-alpes.fr

Csaba Andras Moritz is with the Department of Electrical and Computer Engineering,University of Massachusetts, Amherst, Massachusetts, USA email: andras@ecs.umass.edu

Morgul and Mircea R. Stan are with the Department of Electrical and Computer Engineering, University of Virginia, Charlottesville, Virginia, USA e-mail: \{mm4uz, mircea\}@ virginia.edu

Dan Alexandrescu is with IROC Technologies, Grenoble, France e-mail: dan.alexandrescu@iroctech.com

*: The work is primarily done when he was with Istanbul Technical University; he is currently affiliated with University of Virginia

This work is part of a project that has received funding from the European Union's H2020 research and innovation programme under the Marie Sklodowska-Curie grant agreement \#691178, as well as supported by the TUBITAK-Career project \#113E760 and TUBITAK-2501 project \#218E068.

Copyright (c) 2020 IEEE. Personal use of this material is permitted. However, permission to use this material for any other other purposes must be obtained from the IEEE by sending a request to pubs-permissions@ieee.org

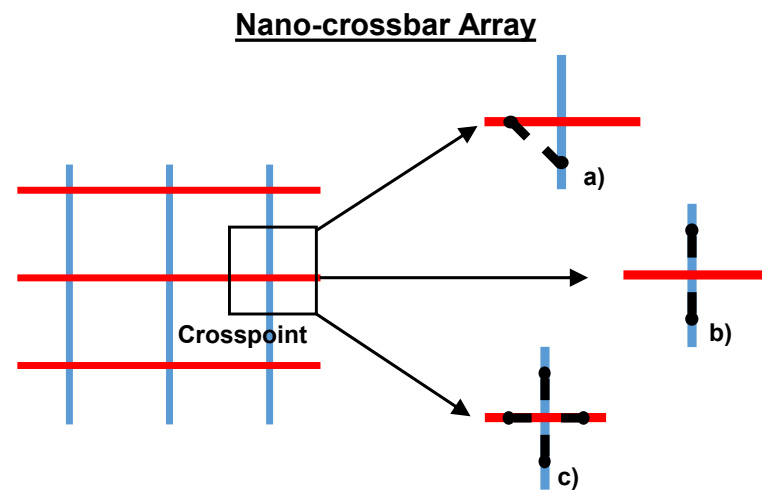

Fig. 1. Switching models of a nano-crossbar array: crosspoint as a) twoterminal switch with terminals in the crossed lines, b) two-terminal switch with terminals in the same line, and c) four-terminal switch.

production. Because of the novel manufacturing techniques, end products have regular and dense forms with area and power efficient structures [15] [4].

Main principle behind crossbar based computing is to utilize crosspoints to behave like switches, either as two-terminal or novel four-terminal [6]. This is illustrated in Figure 1. Depending on the used technology, a two-terminal switch behaves either as a diode [18], a resistive/memristive switch [33] [20], or a field effect transistor (FET) [34]. Diode and resistive switches correspond to the crosspoint structure in Figure 1(a); here, the switch is controlled by the voltage difference between the terminals. Figure 1(b) shows a FET based switch; here, the red line represents the controlling input. This is a unique opportunity that allows us to integrate well developed conventional circuit design techniques into nano-crossbar arrays. Finally, a novel four-terminal switch is demonstrated in Figure 1(c). Four terminal architecture has either all of its terminals connected or disconnected. The desired state is actualized with a controlling input, which is not present in the Figure 1(c) and has a separate physical formation from the crossbar which is thoroughly explained for different technologies in [6] [30]. Detailed TCAD simulations and technology development are presented in [30]. In addition, a realization with standard CMOS process is demonstrated in [13].

Contrary to the conventional technologies, circuit design steps of nano-crossbar arrays are not fully incorporated due to their emerging nature. Motivated by this, we expand and update our preliminary integrated synthesis methodology in [24], and present it in length. Main steps of the methodology 


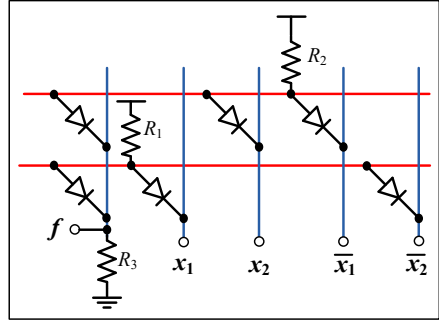

a)

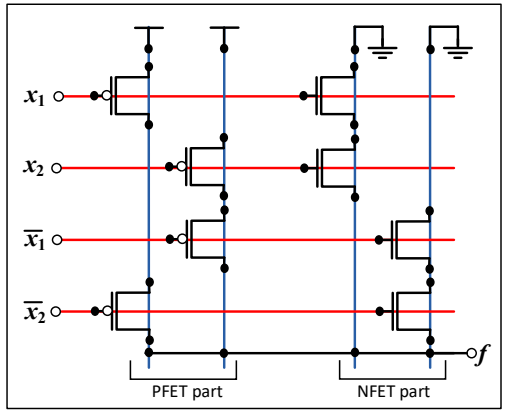

c)

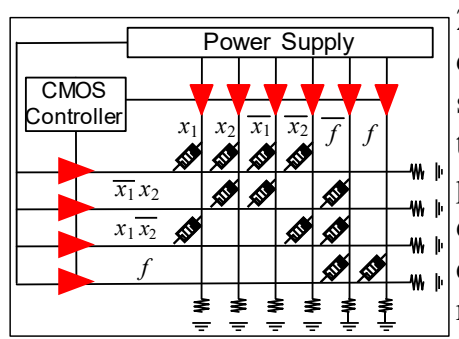

b)

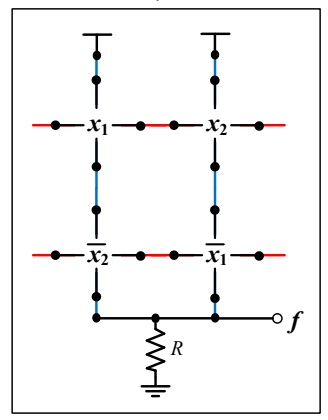

d)
Fig. 2. Realization of $f_{X O R_{2}}$ with different nano-crossbar types: crosspoint as a) diode, b) memristor, c) FET, and d) four-terminal switch.

are logic synthesis, defect/fault tolerance and performance optimization. In Section II, overview of the methodology is stated with the background information. Details of the design steps are stated in the following sections III IV V. We present a case study to elaborate the methodology conclusively in section VI. For sake of clarity, the significant experimental results are shown in the related sections. Main contributions are as follows:

- Expanded version of integrated synthesis methodology presented in [24].

- Multi-output logic synthesis for four-terminal lattices and comparison with others.

- A greedy optimization algorithm for two-level singleoutput memristor crossbar (logic synthesis).

- Defect tolerance technique for four-terminal lattice.

- Performance (delay-power) analysis of diode, FET and four-terminal arrays.

\section{OVERVIEW OF CiRCUit DESIGN STEPS}

\section{A. Background}

Nano-crossbar arrays are first shown to be realizable conceptually in [33], [36] and then physically in [46], [48] as an integrated circuit. After that, research mostly follows the order of technology demonstration such as logic synthesis with ideal (non-defective) arrays, logic synthesis with defective arrays, performance-aware design (performance analysis/optimization), and technology development. This study targets to integrate the current researches with completing missing parts.

1) Logic Synthesis: Main goal of logic synthesis process is to optimize the area size of the circuit through formalizing the circuit size specific to underlying technology. To illustrate different approaches, we show examples for the realization of $f_{X_{O R}}=x_{1} \overline{x_{2}}+\overline{x_{1}} x_{2}$ in Figure 2. Logic synthesis models for diode and memristor based crossbars are quite similar to Programmable Logic Array (PLA) as can be seen in Figure 2(a) and 2(b). Memristor based crossbars have one major difference that establishing the output goes through several states/loops (for further information refer to [44]). We chose the approach in [44] for the memristive crossbars, because the proposed architecture design does not lose its functionality due to the sneak path issues [20]. For FET based crossbars, each logic function product and dual function product is realized by a separate column, as seen in Figure 2(c). Each input is assigned to a row for controlling all the FETs on corresponding row. Finally, a four-terminal based crossbar; here every crosspoint performs switching on all four directions and connection between top and bottom yields 1 as output and 0 otherwise. Control lines of crosspoints are not shown in Figure 2(d), and detailed explanation of control lines can be found in [6] [30].

2) Defect/Fault Tolerance: Anticipated fault rates are much higher for nano-crossbars, as expected, compared to those of conventional CMOS circuits [35]. Therefore, during logic mapping, consideration of faults is mandatory. This applies to the all technology types such as diode, FET based or novel four-terminal based arrays. In short, contrasting the conventional CMOS approach, certain switches cannot be used in mapping process, therefore mapping procedure becomes an assignment problem. Early attempts to overcome this issue consists of locating a fault-free region so no crossbar specific assignment is necessary as in [37]. However, it has quickly become apparent that fault free region is not satisfactory in terms of area size [41]. For this reason, researchers focus on challenges including defect and variance tolerances [40] [28]. In this study, we apply a new fault tolerance technique for four-terminal crossbars (lattices).

3) Performance Optimization: With the process variability data (as an extended concept of defects) of crossbars, performance-aware design can be accomplished. Given that targeted technologies have different performance characteristics, to perform fair comparison, their dependencies on target function should be analyzed. Previously, we have analyzed and extracted delay and power characteristic with dependencies on given function for memristor crossbars [43]. In this study, we present delay and power model for diode, FET, and fourterminal lattice. These models characterize the performance of crossbar technologies for given target function. Idea is to optimize a crossbar with given design specification. However, with the lack of experimental results, optimization and comparison studies are limited with modeled characteristics.

\section{B. Overview of Integrated Methodology}

As briefly stated, nano-fabrication produces switching nanocrossbar arrays with varying properties, structurally and/or component-wise. These random characteristics need to be considered carefully by the synthesis process. For example, a competent methodology must regard basic technology preference for switching elements, and defect or fault rate of the given nano-crossbar. Presented synthesis methodology in this study comprehensively covers the all specified factors and provides optimization algorithms for each step of the process. The diagram, given in Figure 3, summarizes the methodology with demonstrating every step including annotated research tasks. 
Nano-crossbar Array Integrated Synthesis Methodology

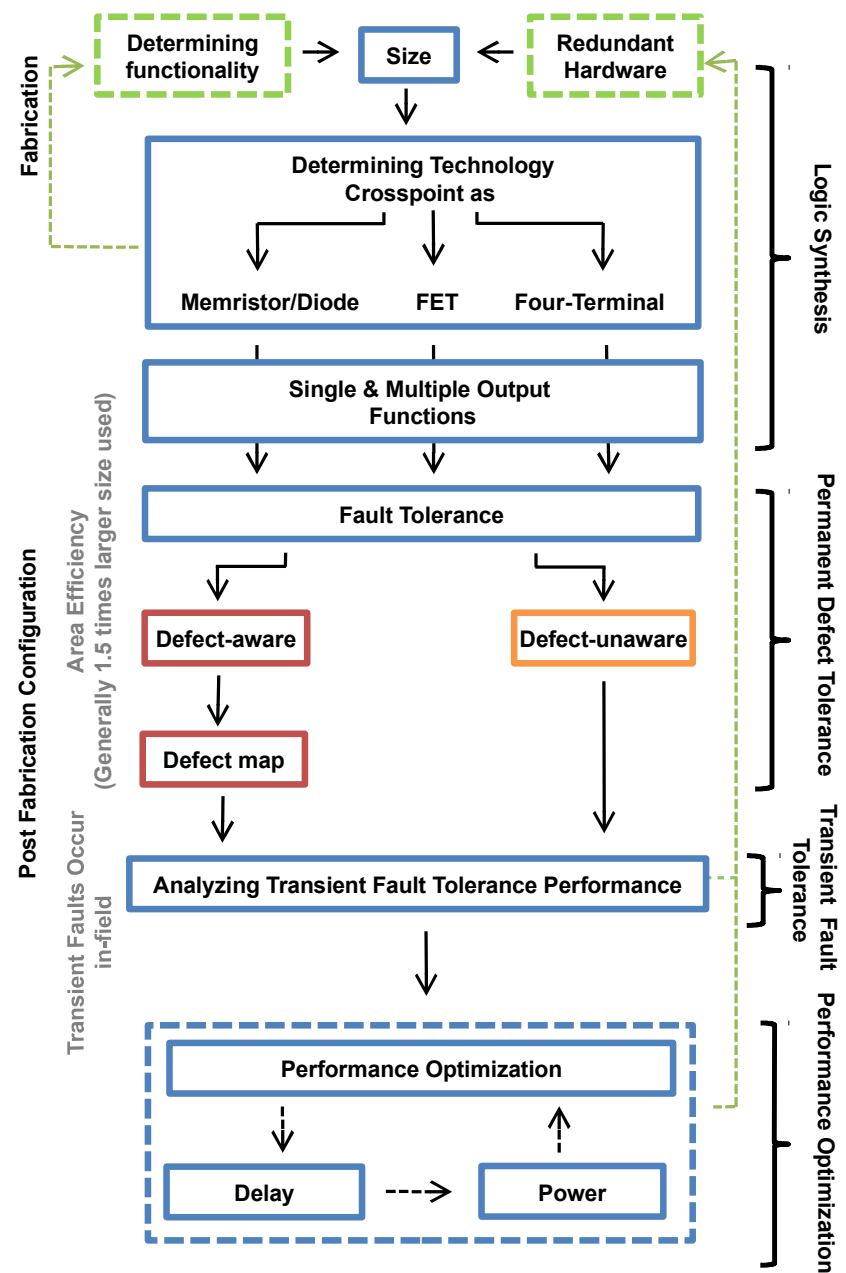

Fig. 3. Integrated synthesis methodology scheme for nano-crossbar arrays

The first step (logic synthesis) covers the decision of switching technology and generate logic function description in crossbar form. The main goal is to determine which of the diode/memristor, FET, or four-terminal based components are to be used. This step is one of the most important procedures determining the area size of the nano-crossbar that is the chief optimization metric. Logic synthesis with diode/memristor, FET, and four-terminal switching technology is in Section III.

The second step (fault tolerance) covers the permanent faults (defects forming in the course of fabrication) and the transient faults (which occur in-field). The main goal is to obtain a valid mapping of a given logic function in crossbar form, produced by logic synthesis step, and defect map. There are two distinct approaches titled as defect-aware and defect-unaware. The first approach employs faults existing in nano-crossbar during the mapping ofthe function, hence the name aware. The second approach avoids the defects by attempting to find a defect-free region on the nano-crossbar at the beginning, so that the mapping of the logic function is straightforward. Finally, transient fault analysis is performed with respect to fault rates/types and fed back to logic synthesis step. Detailed explanations are given in Section IV.

The third step (performance optimization ) covers final delay and power issues. The main goal is to analyze delay and power consumption of arrays by showing their dependencies on the properties of a given logic function as well as specifics of the used technology. Detailed explanations are given in Section $\mathrm{V}$.

To demonstrate the whole process in work, a case study is provided to elucidate the proposed synthesis mechanism in Section VI.

\section{LOGIC SYNTHESIS}

At the start, crossbar (or array used interchangeably) switching technology, i.e., diode, memristor, FET, or four-terminal, needs to be chosen based on the following criteria:

- Crossbar size (number of rows and columns)

- Number of outputs (single or multiple function realization)

- Fabrication complexity

- Power and delay specifications

- Application requirements

A decision can be made on the importance and priority of the listed items, depending on the preference. For example, if an application demands in-memory computing, then the memristor technology can be chosen for the realization of logic functions, since the memristor can also be used as a memory unit.

On the other hand, the realization of a logic function using a diode or a memristor based crossbar requires less number of crosspoints than those of the FET based crossbars. However, the FET based designs consume less power than the diode/memristor-based design. Moreover, the four-terminal based crossbar includes less number of crosspoints than other crossbar designs [26].

Initially, we examine the logic synthesis techniques developed for diode, memristor, and FET based crossbars in the literature and then we formulate their crossbar sizes required to implement given logic functions. We also present results for four-terminal switch based arrays to synthesize multi-output functions.

In the second part, we present a new two-level synthesis technique for memristor based crossbars and compare them with other techniques in the literature.

\section{A. Area Comparisons for Different Crossbar Technologies}

We present the Logic Synthesis step of the integrated methodology, considering only the number of crosspoints in the crossbar. The size of a crossbar array including diode, memristor, FET, and four-terminal is given as follows:

- Diode: (\# of products of all $\left.\left.f_{i}\right)+n\right) \times$ $((\#$ of literals in $f)+n)$

- Memristor: ((\# of products of all $\left.\left.f_{i}\right)+n\right) \times$ $((\#$ of literals in $f)+2 n)$ [worst-case]

- FET: (\# of literals in $f+n) \times$ ((\# of products of all $\left.f_{i}\right)+$ (\# of products of all $\left.f_{i}^{D}\right)$ )

- Four-terminal: (largest of \# of products \# in $f_{i}^{D} s$ ) $\times\left(() \#\right.$ of products of all $\left.\left.f_{i}\right)+n-1\right)$ [worst-case]

where $n$ is the number of logic functions (the number of outputs); $f_{i}$ denotes the $i^{t h}$ logic function, and $f_{i}^{D}$ stands for its dual with $1 \leq i \leq n$. 
TABLE I

Array Size Comparison of Diode, Memristor, FET, and FoUR-TERMINAL SWITCH BASED ARRAYS ON MULTIPLE OUTPUT FUNCTIONS

\begin{tabular}{|c|c|c|c|c|}
\hline Benchmarks & Diode & Memristor & FET & Four-Terminal [2] [1] \\
\hline squar5 & 594 & 884 & 900 & $\mathbf{1 0 8}$ \\
\hline rd53 & 442 & 560 & 819 & 120 \\
\hline misex1 & 437 & 600 & 1334 & $\mathbf{1 2 6}$ \\
\hline sqrt8 & 840 & 1032 & 1340 & 165 \\
\hline b12 & 2028 & 2544 & 3003 & $\mathbf{1 8 0}$ \\
\hline inc & 897 & 1280 & 2231 & 235 \\
\hline bw & 1900 & 3300 & 1824 & $\mathbf{4 0 5}$ \\
\hline sao2 & 1488 & 1764 & 3672 & 476 \\
\hline rd73 & 2210 & 2620 & 4658 & 606 \\
\hline clip & 2875 & 3528 & 6256 & 685 \\
\hline rd84 & 5180 & 6240 & 10960 & 2320 \\
\hline ex5p & 10823 & 19596 & 32864 & 2664 \\
\hline ex1010 & 8820 & 11800 & 57960 & 5958 \\
\hline apex4 & 16835 & 25480 & 72335 & 7308 \\
\hline
\end{tabular}

As mentioned in Section I, the logic synthesis on diode and memristive based crossbars is similar to the PLA like synthesis. Thus, the techniques, such as product sharing and phase changing used in the PLA design, are also applicable in these designs. Since, the array sizes can be further reduced using the product sharing, these array size formulations can be considered as an upper bound for the logic synthesis techniques.

For the single and multiple output function realization, the synthesis methodology for FET crossbar does not allow us to produce multi-level logic synthesis, only two-level approach can be used [35]. However, multi-level logic synthesis approach is feasible for the diode and memristive crossbars [42]. Therefore, the optimization of array size still demands further research for the diode and memristor based designs.

The logic synthesis using four-terminal crossbars, generally known as switching lattices, is a new method. As shown in [6], Altun presented a useful logic synthesis technique for the switching lattices. However, this method cannot find the optimal solution in terms of the lattice size. Therefore, new specific logic synthesis methodologies are needed to be presented. As shown in [17] and [26], optimal synthesis methodologies are provided. Moreover, decomposition based approximate techniques are presented such as XOR based [25] [9], p-circuit [10], and dimension reducibility [11] decompositions. Furthermore, a very efficient (20x faster) technique for very large functions is recently developed to make logic synthesis of four-terminal nano-crossbar arrays more feasible [3].

However, all of these studies focus on the realization of a single logic function using switching lattices. On the other hand, in [2] [1], three main steps are presented to realize the multiple functions using switching lattices. These steps are given as follows: 1) find the realization of each logic function using a switching lattice; 2) merge these lattices into a single lattice; 3 ) check if these lattices can be realized using a smaller number of rows and columns such that the final lattice includes a small number of four-terminal switches.

This article is the first to present the sizes of diode, memristor, FET, and four-terminal based crossbar on the multiple output functions. The results are given in Table I. Note that the
TABLE II

AREA COMPARISON OF TWO-LEVEl LOGIC SyNTHESIS ALGORITHMS INITIAL GREEDY ALGORITHM (GA-INITIAL) AND PROPOSED GREEDY Algorithm (PGA) With OPTIMAL (BRUTE FORCE) AND BASIC [44] APPROACHES FOR MEMRISTIVE ARRAYS

\begin{tabular}{|c|c|c|c|c|}
\hline Benchmarks & Basic [44] & GA-initial [43] & PGA & Optimal \\
\hline rd53 & 560 & 416 & 416 & 416 \\
\hline squar5 & 884 & 858 & 832 & 780 \\
\hline inc & 1280 & 1280 & 1280 & 1248 \\
\hline rd73 & 2620 & 2620 & 1940 & 1940 \\
\hline misex1 & 600 & 750 & 600 & 600 \\
\hline sqrt8 & 1032 & 648 & 648 & 648 \\
\hline ex5p & 19596 & 19312 & 19312 & $* *$ \\
\hline rd84 & 6240 & 6072 & 4584 & 4584 \\
\hline clip & 3528 & 3500 & 3388 & 3332 \\
\hline sao2 & 1764 & 1176 & 1372 & 1176 \\
\hline ex1010 & 11800 & 11800 & 11800 & 11800 \\
\hline alu4 & 25696 & 16544 & 16544 & 16192 \\
\hline b12 & 2544 & 1872 & 1776 & 1776 \\
\hline table5 & 11136 & 11136 & 11136 & $* *$ \\
\hline vg2 & 7854 & 7854 & 7854 & 7854 \\
\hline ** Time exceeds 600 seconds
\end{tabular}

results given in bold under the four-terminal column indicates that they are found using the approximate algorithm of [2] [1] (JANUS), following the three steps described above. On the other hand, the other results are found using a divide and conquer method, based on the divide and synthesize (DS) method of [2] [1], following the first two steps described above.

As can be observed from Table I, the four-terminal based crossbar arrays include significantly less number of crosspoints when compared to the diode, memristor, and FET based crossbar arrays.

For the logic synthesis of memristor based crossbars, there exists more area efficient techniques than in [44]; such as [8] (SIMPLER MAGIC ), [38], or [31] (which are essentially improved versions of the MAGIC [21]). For example, misex 1 can be realized in an array which has the size of 33,88 and 52 with [8], [38] and [31], respectively. However they require much larger number of operation cycles: 83, 52 and 31 with [8], [38] and [31], respectively. Note that, required number of cycles is only 7 for [44]. This number can even be reduced to 5 with the proposed technique [43]. On the other hand, as stated in [45], the method in [44] can support the method MAGIC. Furthermore, since the method of MAGIC (and improved versions of it) uses all crosspoints in the array (density is $100 \%$ ), its defect intolerance is much higher than [44]. Therefore, we used the approach in [44] and implemented the improved version to our integrated synthesis methodology.

\section{B. Proposed Greedy Algorithm for two-level multi output} synthesis for Memristive Arrays

In memristive crossbar arrays, function outputs and their negations are produced [44]. However production order changes the array size. We have called phase_combination-0 and phase_combination-1 realization, if realization is happened based on an output itself and its negation, respectively. For instance, for the realization of phase_combination1 , first its negation is produced [43] and at last original function outputs. Based on this property, array size of memristive crossbars can be optimized. The realization in [44] is phase_combination-1 realization. 


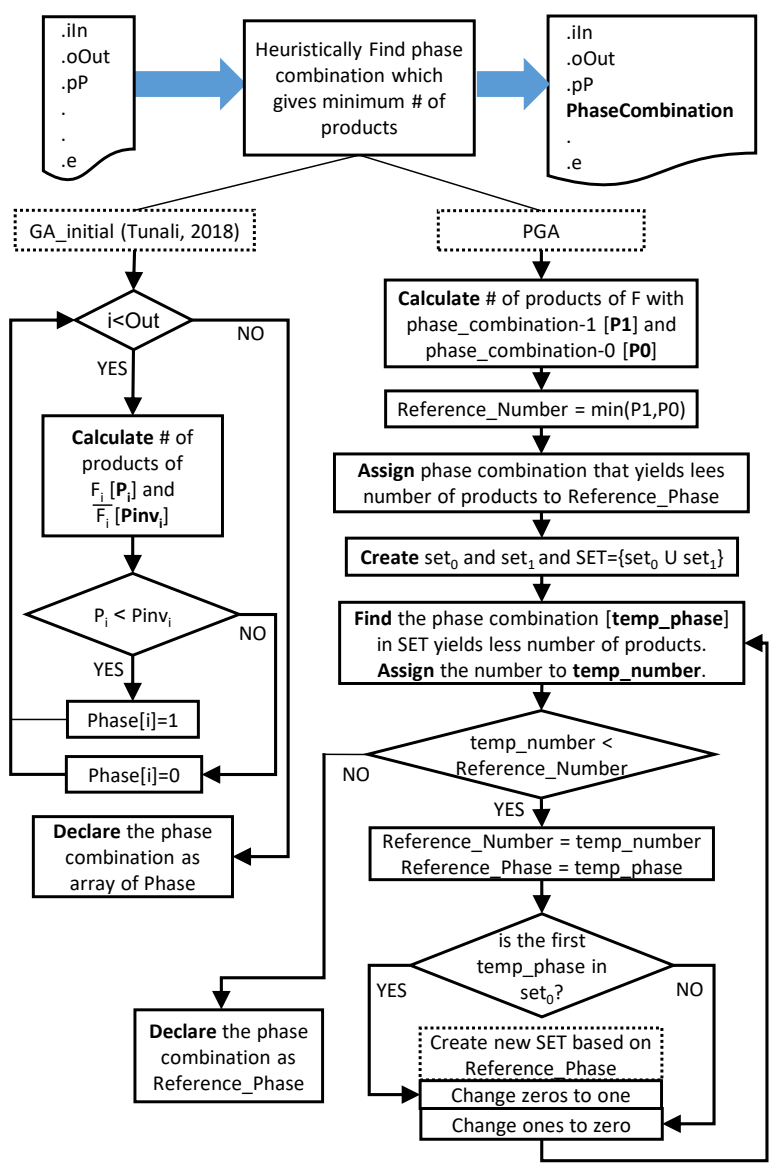

Fig. 4. Block diagrams of two-level multi output logic synthesis algorithms: initial Greedy Algorithm (GA-initial) [43] and Proposed Greedy Algorithm (PGA)

In our previous study [43], we have proposed a greedy algorithm, which only considers outputs individually, and doesn't consider outputs interrelation (i.e. no product sharing at the analysis). We can call this algorithm as "initial Greedy Algorithm (GA-initial)". In this algorithm, only product numbers of individual outputs are considered. On the other hand our new greedy algorithm considers product sharing with the change of phase, meaning it considers total number of products of whole (multi-output) function. We illustrate these two algorithms in Figure 4 side-by-side.

Proposed Greedy Algorithm (PGA): investigates outputs collectively by changing phase of outputs, one at a time, starting from phase_combination- 0 and phase_combination- 1 . Compares them; if any of the changed phase combination has less \# of total product; then keeps that phase and continues searching with changing other outputs' phase one at a time. Algorithm (PGA) is as follows:

1: Calculate and Compare number of products of phase_combination-0 (00..0) and phase_combination-1 (11..1), Assign minimum number of products to Reference_Number, and the phase combination to Reference_Phase,

2: Create two sets of candidate phase combinations; set $t_{0}$ : includes phase combinations that only one output is in "phase-1" and set $_{1}$ : includes phase combinations that only one output is in "phase-0", and Assign $S E T=\operatorname{set}_{0} \cup$ set $_{1}$,
3: Find the phase combinations (temp_phase) which yields minimum number of products (temp_number) for the function in $S E T$.

4: Decide; if temp_number is less than Reference_Number. If YES, Assign temp_number to Reference_Number, and (temp_phase) to Reference_Phase; If NO, Jump to 8 (end of the algorithm),

5: Decide; If the found very first temp_phase is in set $_{0}$,

5a: If YES (in set $t_{0}$ ), Create a new $S E T$, with phase combinations, by changing "0"s of Reference_Phase to "1"s,

5b: If NO (in set $)_{1}$ ), Create a new $S E T$, with phase combinations, by changing "1"s of Reference_Phase to "0"s,

(Note that, every phase combination in $S E T$ has only one alteration with Reference_Phase)

6: Jump to 3,

7: Declare phase combinations as Reference_Phase.

For example, if we are given a function which has three outputs. First we check phases "000" and "111" (phase_combination-0 and phase_combination-1). Then calculate product numbers of phase in phase sets; set $_{0}$ : " 001,010 , and 100" (changing zeros to one), and set $_{1}$ : "110, 101, and 011" (Changing ones to zero) (Note that, we change one at a time). Let's say we compared product numbers and found that phase " 110 " yields the minimum number of products. Then we continue from the phase " 110 ", with changing ones to zero. Means, we check " 100 and 010 " and compare the results with result of phase " 110 ". If there is one which yields less product number chose it, or chose the phase " 110 " for the final phase combination of the function. (Notice that, for a function, which has only three outputs, looking to sets of set $_{0}$ and set $_{1}$ is enough)

To evaluate the algorithms and compare the results, we use espresso and MATLAB ${ }^{\mathrm{TM}}$ on a $3.20 \mathrm{GHz}$ Intel Core i7 CPU with 4GB memory. Results are shown in Table II. Algorithm performances differs from function to function. For six of the examples PGA results less area size than GA-initial. Overall, PGA surpasses GA-initial, yet for two (clip and sao2) of the functions GA-initial results less area size than PGA. For the rest they produce the same result. GA-initial's major advantages is that it has almost no time cost. It can be used for any case.

\section{Defect/Fault Tolerance}

In this section, we investigate the effects of permanent and transient faults on the behavior of a logic function mapped on a given crossbar array. We categorize faults into 2 classes: permanent, generated by physical defects, and transient faults. As mentioned in Section I, actual nano-crossbar arrays being fabricated with emerging technologies, are affected mostly by defects, due to the poor technology yields. Indeed, immature process technologies used for nanowires, memristive, etc. elements generate high defect density crossbars. At the same time, these elements also show instabilities and variabilities (behaving like transient or intermittent faults) and they are also affected by transient phenomena occurring in the field. 


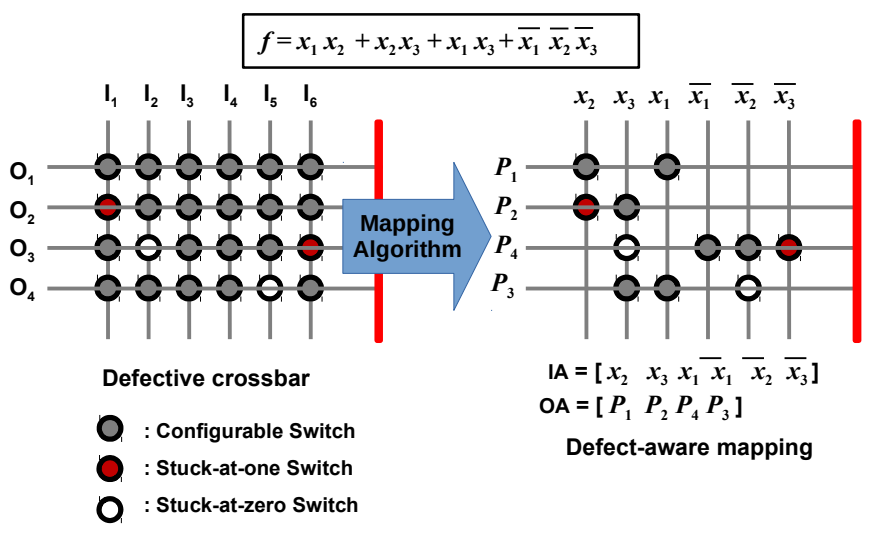

Fig. 5. Nano-crossbar array with faulty/defective crosspoints and mapping process.

Permanent fault (defect) tolerance, also called defect avoidance, basically means finding defect-free regions or defect free crosspoints that can still be employed during logic mapping, and it is usually done by the logic function remapping (at the algorithm level) and/or by reconfiguration, at algorithm or hardware level. Permanent fault model can be seen in Figure 5 demonstrating only stuck-at-0 (open) and stuck-at-1 (close) faults as being the most representative permanent faults observed. This categorization is based on actual physical realization of nano-crossbar array as reported in [22] and [12] which state that fault rates reach up to $10 \%$ and the most common faults are stuck-at-0 (open).

Transient and intermittent faults, on the other hand, manifest themselves due to particular combinations of topological, environmental factors or process mismatches and instabilities. They can be tolerated by proper architectural reconfiguration, but also by instability and variation-aware design. That is to say by using properly sized, or well estimated hardware redundancy in term of spare cells, critically sensitive cells can be circumvented or protected. Transient fault model can have multiple forms meaning they can be seen as parasitic, 0to-1 (1-to-0 ) transitions, parasitic positive (negative) pulses, neighbor cells pattern dependent transitions, etc. Transient fault domain is very closely related to a specific technology. Transient faults are more efficiently covered by fault tolerance strategies, as fault avoidance techniques will yield to a much higher area, but also due to the complexity of the technology dependence analysis.

For both type of faults, it is mandatory to perform crossbar sensitivity analysis as the mapping algorithm can be enhanced with fault avoidance properties, and also can be used to further drive the fault tolerance strategy.

\section{A. Defect Tolerance for Diode, Memristor and FET}

Defect tolerance is achieved by mapping a target logic function on a defective crossbar using a distinct input and output assignment. This problem is considered as NP-complete [32]. For the worst-case, $N ! \times M !$ permutations are required to find a successful mapping for $N \times M$ crossbar. Algorithms in the literature use defect-unaware or defect-aware approach.

Defect-unaware algorithms aim to find the largest possible $k \times k$ defect-free sub-crossbar from a defective $N \times N$ crossbar where $k \leq N$. The algorithms are inefficient for

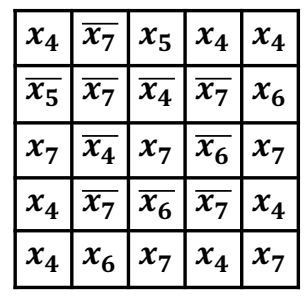

a)

\begin{tabular}{|l|l|l|l|l|}
\hline 1 & 1 & 1 & 2 & 1 \\
\hline 1 & 2 & 1 & 2 & 1 \\
\hline 1 & 2 & 1 & 2 & 1 \\
\hline 1 & 2 & 1 & 2 & 1 \\
\hline 1 & 2 & 1 & 0 & 1 \\
\hline
\end{tabular}

b)

\begin{tabular}{|l|l|l|l|l|}
\hline 1 & 0 & 1 & 0 & 0 \\
\hline 1 & 0 & 1 & 1 & 1 \\
\hline 1 & 2 & 0 & 2 & 2 \\
\hline 0 & 1 & 1 & 0 & 0 \\
\hline 0 & 2 & 2 & 2 & 0 \\
\hline
\end{tabular}

c)
Fig. 6. a) Lattice design for the example function $f$ and its sensitivity map for b) SAO and c) SA1.

high fault rates - obtained $k$ values are much smaller than $N$. In this regard, defect-aware algorithms perform much more satisfactorily [40]. Detailed analysis of both approaches can be found in [41].

Defect-aware algorithms consider the defect characteristics (stuck-at-0 or stuck-at-1), then decide which switch to employ during the mapping. In our previous work [40], we have proposed an efficient heuristic algorithms which aims to match defective crossbar and the given logic function. For this, it denotes crossbars as matrices. Therefore, it can perform sorting, matching and backtracking steps efficiently. It makes repetition for a limit of permutation. This controls heuristic feature of the algorithm.

Defect tolerance for nano-crossbars is a well established field with numerous research papers and for this reason we focus on four-terminal architectures which is, to our knowledge, exclusive to this study.

\section{B. Defect Tolerance for Four Terminals Devices}

Four-terminal defect tolerance demands a different approach than the architectures we have covered so far. For this reason, we present a novel method, which is firstly introduced in this paper. The proposed method utilizes a prior sensitivity analysis of crossbar (latttice) to specify critical switches, and strengthens them with proposed mitigation factors. The same naming conventions are applicable, regarding defects which are categorized as stuck-at-0 (SA0) and stuck-at-1 (SA1), called Stuck at Fault Model (SAF). Furthermore, we describe a new model that can be considered for crossbars (lattices), that consists in changing a switching literal in a cell $c_{i, j}$ of the lattice with a literal that is in a adjacent cell (i.e., $c_{i-1, j}, c_{i+1, j}, c_{i, j-1}$, or $\left.c_{i, j+1}\right)$. We denote this fault model as Adjacent Cellular Fault Model (ACF). In addition, we follow the same terminology adopted in [6] and [17] by addressing crossbar as lattice and switch as cell to be consistent and emphasize the distinction of four-terminal approach. Finally, it should be noted that as opposed the previous sections, we provide a more detailed explanation due to original technical contribution presented in this section.

1) Defect Injection Methodology: The two fault injections SAF and ACF are repeated for each cell of the lattice. Once a "defective" lattice is obtained, both algorithms generate all the possible $2^{n}$ inputs (where $n$ is the number of variables). For each input, the simulation algorithms compare the given output with the correct one. The two fault injection algorithms (one for SAF and one for ACF) differ in the calculation of number of defective outputs in the two fault models, as explained in the following subsections. 
Fault Model 1: Stuck-at Faults (SAFs) The sensitivity of the decomposition algorithm on a given lattice is analyzed to face SA0 and SA1, as a model widely adopted today for memristive crossbars. As there is no consensus currently on the fault distribution, we have chosen a uniform distribution for each type of SA0 and SA1 [14]. The fault rates considered may be up to $10 \%$ of the crossbar, all faults being independent, as reported in [14]. The fault injection in the above lattices is performed substituting a single cell with an always stuck-at 1 (SA1) or stack-at 0 (SA0) cell.

Let $E_{i j}^{0}$ (resp., $E_{i j}^{1}$ ), with $1 \leq i \leq r, 1 \leq j \leq s$, be the number of defective outputs with a SA0 (resp., SA1) in the cell $(i, j)$ of the given lattice. Note that $0 \leq\left\{E_{i j}^{0}, E_{i j}^{1}\right\} \leq 2^{n}$. Moreover, when $E_{i j}^{0}$ (resp., $E_{i j}^{1}$ ) is equal to 0 we have that, for any possible input, the lattice output is never changed by the SAF in the cell $c_{i, j}$. In this case, we call the cell $c_{i, j}$ robust w.r.t. SA0 (resp., SA1). Let $R^{0}$ (resp., $R^{1}$ ) be the total number of robust cells w.r.t. SA0 (resp., SA1) in the lattice. Finally, let $E^{0}=\sum_{i=1}^{i=r} \sum_{j=1}^{j=s} E_{i j}^{0}$ (resp., $E^{1}=$ $\sum_{i=1}^{i=r} \sum_{j=1}^{j=s} E_{i j}^{1}$ ) be the total number of defective outputs with SA0 (resp. SA1) in the simulation. For an example of function $f=x_{4} \overline{x_{5}} x_{7}+\overline{x_{4}} x_{6} \overline{x_{7}}+\overline{x_{4}} x_{5} \overline{x_{6}} x_{7}+x_{4} \overline{x_{6} x_{7}}+x_{4} x_{6} x_{7}$ realized in Figure 6(a) (with the method in [6]), in the Figure 6(b) (resp., 6(c) shows the map containing $E_{i j}^{0}$ (resp., $E_{i j}^{1}$ ) in each cell. Consider SA0 case (matrix in Figure 6(b)). Each cell of the matrix contains the number of faulty outputs due to a SA0 in the corresponding cell of the lattice 6(a). When a cell of the matrices from Figures 6(b) (SA0) and 6(c) (SA1), contains the value 0 , the cell of the corresponding lattice is robust. In this example, the overall number of robust cells for the SA0 (resp., SA1) is $R^{0}=1$ (resp., $R^{1}=10$ ).

Fault Model 2: Adjacent Cellular Faults (ACFs) In the classical CFM [16] used for CMOS circuits it is assumed that a fault modifies the behavior of exactly one node $v$ in a given circuit $C$ and that the modified behavior is still combinational. In the case of a switching lattice $L$, the fault model can be described as follows: a cellular fault in $L$ is a tuple $\left(c_{i, j}, l_{c}, l_{f}\right)$, where $c_{i, j}$ is the cell of the lattice $L$ (i.e., fault location), $l_{c}$ is the correct controlling literal in $c_{i, j}$, and $l_{f}\left(\neq l_{c}\right)$ is the faulty controlling literal. We denote adjacent cellular fault a cellular fault where the faulty literal $l_{f}$ is the literal contained in an adjacent cell. More precisely:

Definition 1: Let $l_{h, k}$ be the literal in the cell $c_{h, k}$ of a lattice $L$, with $1 \leq h \leq r, 1 \leq k \leq s$. We have that:

1) A Left Adjacent Cellular Fault (L-ACF) is the cellular fault $\left(c_{i, j}, l_{i, j}, l_{i, j-1}\right)$,

2) A Right Adjacent Cellular Fault $(R-A C F)$ is the cellular fault $\left(c_{i, j}, l_{i, j}, l_{i, j+1}\right)$,

3) A Bottom Adjacent Cellular Fault (B-ACF) is the cellular fault $\left(c_{i, j}, l_{i, j}, l_{i+1, j}\right)$,

4) A Top Adjacent Cellular Fault (T-ACF) is the cellular fault $\left(c_{i, j}, l_{i, j}, l_{i-1, j}\right)$.

For example, consider the lattice depicted in Figure 6(a) and the cell $c_{2,1}$ containing the literal $\bar{x}_{5}$ in the second row from top and first column from left. In this cell we can have three possible adjacent Cellular Faults: 1) R-ACF: $\left(c_{2,1}, \bar{x}_{5}, \bar{x}_{7}\right)$ that makes $\bar{x}_{7}$ the faulty literal of the cell $\left.c_{2,1} ; 2\right) \mathrm{B}-\mathrm{ACF}$ : $\left(c_{2,1}, \bar{x}_{5}, x_{7}\right)$ that makes $x_{7}$ the faulty literal of the cell $c_{2,1}$; 3) T-ACF: $\left(c_{2,1}, \bar{x}_{5}, x_{4}\right)$ that makes $x_{4}$ the faulty literal of the cell $c_{2,1}$. Notice that the cell $c_{2,1}$ cannot be affected by a Left Adjacent Cellular Fault.

Let $E_{i j}^{L}$ (resp., $E_{i j}^{R}, E_{i j}^{B}$, and $E_{i j}^{T}$ ), with $1 \leq i \leq r, 1 \leq$ $j \leq s$, be the number of defective outputs with a L-ACF (resp., R-ACF, B-ACF, and T-ACF) in the cell $c_{i, j}$ of the given lattice. Let $R^{a}$ (with $a \in\{L, R, B, T\}$ ) be the total number of robust cells w.r.t. $a-\mathrm{ACF}$ in the lattice. Finally, let $E^{a}=$ $\sum_{i=1}^{i=r} \sum_{j=1}^{j=s} E_{i j}^{a}$ be the total number of defective outputs with $a-\mathrm{ACF}$ in the simulation.

2) Metrics used for Sensitivity Analysis: Let us consider a lattice with $n$ input Boolean variables, the main aim of our sensitivity analysis is to understand how many inputs combinations (out of all the possible ones, which are $2^{n}$ in number) give an incorrect output value. For this purpose, we inject one error in a cell of the lattice (one error at time), for all cells. This way, we can compute the total number of defective outputs ( $E^{i}$, with $i \in\{0,1, L, R, B, T\}$ ) and the total number of robust cells ( $R^{i}$, with $i \in\{0,1, L, R, B, T\}$ ) as described in the previous section. In order to evaluate the sensitivity of a lattice to SAF and ACF, we propose two metrics. The first one measures the average number of defective outputs face to defect-sensitive cells. The second metric measures the average number of defective outputs in the entire lattice, considering robust and defective, non-robust cells. Note that the total number of cells is the area of the lattice (i.e., $r \cdot s$ ), the number of non-robust cells for SA0 (resp., SA1) is $r \cdot s-R^{0}$ (resp., $r \cdot s-R^{1}$ ), and $2^{n}$ is the total number of possible distinct inputs. Moreover, the number of non-robust cells for $a-\mathrm{ACF}$ (with $a \in\{L, R, B, T\}$ ) is $r \cdot s-R^{a}$.

The sensitivity to defective cells is the total number of inputs that give an uncorrected output $\left(E^{i}\right.$, with $i \in$ $\{0,1, L, R, B, T\})$ divided by the total number of inputs $\left(2^{n}\right)$, for each non-robust cell. The metric can be expressed as: $S_{C}^{i}=E^{i} /\left(2^{n} \cdot\left(r \cdot s-R^{i}\right)\right)$ for each fault $i \in\{0,1, L, R, B, T\}$.

The sensitivity of lattice is the total number of inputs that give an uncorrected output divided by the total number of inputs for each cell: In particular, $S_{L}^{i}=E^{i} /\left(2^{n} \cdot r \cdot s\right)$, with $i \in\{0,1, L, R, B, T\}$.

In summary, the first metric measures the impact of one cell on the probability of failure of a logic function. The second one allows us to evaluate the integrity of the entire lattice and understand if it is possible to map strong, critical functions (for a specific operation) in such lattice, which have high sensitivity to faults or overall high robustness. For instance, if we have a lattice where half of the cells are non robust we can understand that we may not want to use it for critical functions (e.g., control, decision making, etc).

For example, consider the lattice depicted in Figure 6(a) and the map in Figure 6(c) showing the values of $E_{i j}^{1}$ in each cell in the SA1 model. Note that the number of rows and columns is $r=s=5$ and the number of different variables in the lattice is $n=4$ (i.e., $x_{4}, x_{5}, x_{6}$, and $x_{7}$ ). Moreover, the total number of incorrect outputs is the sum of all the errors, i.e., $E^{1}=21$ and the number of robust cells (i.e., cells with no 
errors) is $R^{1}=10$. Therefore, the sensitivity of defective cells is $S_{C}^{1}=E^{1} /\left(2^{n} \cdot\left(r \cdot s-R^{1}\right)\right)=21 /\left(2^{4} \cdot(5 \cdot 5-10)\right)=$ $21 / 240$. This means that out of all possible errors, which are 240 in number, we have 21 errors. The second metrics does not take into account the robust cells, therefore we have that $S_{L}^{1}=E^{1} /\left(2^{n} \cdot(r \cdot s)\right)=21 /\left(2^{4} \cdot(5 \cdot 5)\right)=21 / 400$.

3) Benchmarks and Simulations: The defect simulations have been run on a machine with two Intel Xeon E5-2683 for a total of 64 CPUs and 756 GByte of main memory, running Linux CentOS 7. The benchmarks functions are expressed in PLA form and are taken from a subset of LGSynth93 [47]. Each output of a function is implemented as a separate Boolean function for total of about 1000 functions.

The software used for simulations is written in $\mathrm{C}++$. We used ESPRESSO [23] to implement the method described in [6], and a collection of Python scripts for computing minimum-area lattices by transformation to a series of SAT problems, to simulate the results reported in [17].

In Table III, we report a sample of benchmark functions and their sensitivity values, according to the metrics presented before. In particular, Table III refers to lattice synthesized as described in [6] and [17]. The benchmarks marked with $a \star$ in Table III, were stopped after ten minutes of SAT execution and, for this reason, they are missing from the benchmarks synthesized using quantified Boolean logic.

More precisely, in both methods, the first column reports the name and the number of the considered outputs of each function. The following columns report dimension $(r \times s)$ required for the synthesis of a given function according to each decomposition method, and the number of input variables $n$. Columns from 4 to 11 refers to Stuck At fault model, columns from 12 to 27 to Adjacent Cellular Fault model showing the total number of errors $E$, the Sensitivity of defective cells $S_{C}$, the Sensitivity of lattice $S_{L}$ and the percentage of robust cells $\% R / r \times s$. Table III shows that the synthesis method proposed by [6] produces lattices with a lower number of errors and a higher number of robust cells. However, the area of these lattices is greater than the area of the corresponding lattices synthesized using [17]. For example, consider the benchmark circuit alu3(2): the area of the lattice minimized with [6] (resp., [17]) method is $10 \times 11$ (resp., $6 \times 4$ ), while the percentage of robust cells in the lattice synthesized using [6] (resp., [17]) method is $98 \%$ (resp., 33\%) in the SA0 case. For the other fault models the percentage's comparison is similar.

Table IV describes the overall results for the considered benchmarks. More precisely in the columns from one to three is reported the synthesis method, the average lattice area, and the average number of input. Columns from four to twentyone show the average values of $S_{C}, S_{L}$, and $\% R / r \times s$ for each fault injection methodology. The columns from twentytwo to twenty-four contain the average value of $S_{C}, S_{L}$, and $\% R / r \times s$ obtained considering all the fault models.

Table IV shows that the percentage of cells that are considered robust according to our metrics is higher in the first approach [6]. For example if we consider the benchmark prom 2(0) with SA1 faults, using the approach [6] we have $100 \%$ of resilient cells, instead using the approach [17] there are no resilient cells. This is due to the more constrained structure of the lattices produced by the first synthesis method. Indeed, the method proposed in [6] computes a lattice for $f$ and its dual, which it is in general less compact than the lattice given by [17]. However, we can note that the sensitivity of the lattices is quite low for both methods. In fact, the experiments show that, in general, non-robust cells compute a failed output for a very limited number of inputs. In particular, lattices present lower sensitivity to Adjacent Cellular faults, with respect to the Stuck-At faults. This is due to the fact that when two adjacent cells contain the same value, this situation will not show an impact on the output. In both synthesis approaches, we observe that the lattice is more sensitive to SA0 faults, while in the case of SA1 faults, it requires higher percentage of redundant cells.

It is worth noting that we test lattices with up to 12 variables, this makes interesting the use of this technology to implement multipliers, or other complex arithmetic functions.

4) Mitigation by Defect Avoidance: From the above results, it can be seen that the two analyzed mapping algorithms show different sensitivities of the output of a given function. As a matter of fact, the more restrictive an algorithm in terms of area it is (closer to the optimal solution), the higher the defect sensitivity of the output to a cell defect. It is thus mandatory to include the mapping algorithm defect-avoidance heuristics, but hardware-level defect tolerant scheme may also be necessary, especially in the case of very high defect densities and transient faults rates. Hardware redundancy schemes can be used at the lattice column level, or at the block level [27], as the basic computation unit of a memristor array is not the memristive cell, as in classical CMOS based memories, but the entire column. Therefore, several redundant columns or blocks can be added to the initial design to replace the memristive RAM affected columns but also to overcome the situation when potential SAFs affect the redundant parts as well.

Besides Redundancy Repair Techniques, other defect tolerance techniques can be used. For example, Error Correcting Codes (ECC) especially when transient faults are the main issue, but also fault masking techniques to typically repair permanent faults, or combinations of both. Various advanced error correction techniques have been proposed for emerging technologies structures, which provide improvements over the conventional ECC or Redundancy Repair techniques [19]. But it is worth noting that all of them are generating quite significant area overhead due to the encoding/decoding circuitry, or to the extra spare rows and columns, or a more complex addressing and accessing schemes, but they also impact the write/read margins of the lattice, and increase the access latency, and power consumption. Therefore, a combination of efficient defect avoidance mapping algorithm combined with Redundancy based Repair techniques and/or ECC codes will lead to fully functional lattices even for high defect and transient fault rates. Mapping of logic functions on crossbar arrays are thus divided into two main phases: mapping phase to write the parameters of functions in the memristive array and a read operation to check the results from the crossbar. 
TABLE III

A SAMPLE OF BENCHMARK FUNCTIONS SYNTHESIZED WITH [6] AND [17] APPROACHES AND THEIR SENSITIVITY VALUES

\begin{tabular}{|c|c|c|c|c|c|c|c|c|c|c|c|c|c|c|c|c|c|c|c|c|c|c|c|c|c|c|}
\hline \multirow{2}{*}{ name } & \multicolumn{2}{|c|}{ Benchmark Size } & \multicolumn{8}{|c|}{ Stuck at Fault Model } & \multicolumn{16}{|c|}{ Adjacent Cellular Fault Model } \\
\hline & $r \times s$ & $n$ & $E^{0}$ & $S_{C}^{0}$ & $S_{L}^{0}$ & $\% \frac{R^{0}}{r \times s}$ & $E^{1}$ & $S_{C}^{1}$ & $S_{L}^{1}$ & $\% \frac{R^{1}}{r \times s}$ & $E^{R}$ & $S_{C}^{R}$ & $S_{L}^{R}$ & $\% \frac{R^{R}}{r \times s}$ & $E^{L}$ & $S_{C}^{L}$ & $S_{L}^{L}$ & $\% \frac{R^{L}}{r \times s}$ & $E^{T}$ & $S_{C}^{T}$ & $S_{L}^{T}$ & $\% \frac{R^{T}}{r \times s}$ & $E^{B}$ & $S_{C}^{B}$ & $S_{L}^{B}$ & $\% \frac{R^{B}}{r \times s}$ \\
\hline \multicolumn{27}{|c|}{ Synthesis with Dual Method [6] } \\
\hline alu3(2) & $10 \times 11$ & 8 & 16 & 0.008 & 0 & 96 & 4 & 0.008 & 0 & 98 & 12 & 0.003 & 0 & 87 & 12 & 0.003 & 0 & 87 & 4 & 0.001 & 0 & 89 & 4 & 0.001 & 0 & 89 \\
\hline b12(6) & $9 \times 6$ & 9 & 160 & 0.028 & 0.006 & 80 & 2072 & 0.225 & 0.075 & 68 & 80 & 0.013 & 0.003 & 78 & 72 & 0.013 & 0.003 & 80 & 2072 & 0.150 & 0.075 & 50 & 344 & 0.037 & 0.012 & 67 \\
\hline $\mathrm{br} 2(0)^{\star}$ & $5 \times 14$ & 12 & 227 & 0.002 & 0.001 & 50 & 546 & 0.003 & 0.002 & 41 & 100 & 0.001 & 0 & 56 & 125 & 0.001 & 0 & 54 & 545 & 0.003 & 0.002 & 36 & 381 & 0.002 & 0.001 & 41 \\
\hline $\operatorname{clpl}(3)$ & $6 \times 6$ & 11 & 4085 & 0.074 & 0.055 & 25 & 1382 & 0.042 & 0.019 & 57 & 1010 & 0.019 & 0.014 & 28 & 3342 & 0.063 & 0.045 & 28 & 1381 & 0.032 & 0.019 & 42 & 274 & 0.008 & 0.004 & 53 \\
\hline $\operatorname{dist}(2)^{\star}$ & $33 \times 30$ & 8 & 301 & 0.005 & 0.001 & 78 & 207 & 0.007 & 0.001 & 89 & 182 & 0.004 & 0.001 & 84 & 198 & 0.004 & 0.001 & 82 & 187 & 0.005 & 0.001 & 86 & 175 & 0.005 & 0.001 & 85 \\
\hline jbp(44) & $2 \times 10$ & 11 & 576 & 0.031 & 0.014 & 55 & 1024 & 0.071 & 0.025 & 65 & 192 & 0.007 & 0.005 & 35 & 64 & 0.003 & 0.002 & 45 & 640 & 0.045 & 0.016 & 65 & 1024 & 0.056 & 0.025 & 55 \\
\hline $\operatorname{luc}(13)$ & $9 \times 10$ & 6 & 70 & 0.024 & 0.012 & 50 & 36 & 0.019 & 0.006 & 68 & 36 & 0.017 & 0.006 & 62 & 38 & 0.017 & 0.007 & 61 & 29 & 0.014 & 0.005 & 64 & 36 & 0.014 & 0.006 & 57 \\
\hline $\mathrm{m} 4(7)^{\star}$ & $32 \times 29$ & 8 & 514 & 0.006 & 0.002 & 63 & 278 & 0.010 & 0.001 & 89 & 354 & 0.005 & 0.001 & 71 & 341 & 0.005 & 0.001 & 71 & 271 & 0.008 & 0.001 & 85 & 276 & 0.007 & 0.001 & 85 \\
\hline $\max 128(9)$ & $10 \times 9$ & 7 & 188 & 0.029 & 0.016 & 43 & 51 & 0.025 & 0.004 & 82 & 135 & 0.022 & 0.012 & 46 & 134 & 0.024 & 0.012 & 52 & 43 & 0.015 & 0.004 & 76 & 51 & 0.015 & 0.004 & 71 \\
\hline prom2(0) & $8 \times 6$ & 8 & 122 & 0.063 & 0.014 & 78 & 184 & 0 & 0 & 100 & 92 & 0.043 & 0.009 & 80 & 92 & 0.039 & 0.007 & 83 & 168 & 0 & 0 & 94 & 136 & 0 & 0 & 94 \\
\hline $\operatorname{radd}(3)^{\star}$ & $36 \times 36$ & 8 & 471 & 0.012 & 0.001 & 88 & 388 & 0.017 & 0.001 & 93 & 314 & 0.009 & 0.001 & 89 & 284 & 0.008 & 0.001 & 88 & 384 & 0.013 & 0.001 & 91 & 334 & 0.011 & 0.001 & 91 \\
\hline $\operatorname{rd} 73(1)^{\star}$ & $42 \times 42$ & 7 & 299 & 0.008 & 0.001 & 83 & 178 & 0.008 & 0.001 & 90 & 159 & 0.006 & 0.001 & 89 & 171 & 0.006 & 0.001 & 88 & 178 & 0.006 & 0.001 & 88 & 171 & 0.006 & 0.001 & 88 \\
\hline \multicolumn{27}{|c|}{ Synthesis with Quantified Boolean Logic [17] } \\
\hline alu3(2) & $6 \times 4$ & 8 & 398 & 0.097 & 0.065 & 33 & 168 & 0.041 & 0.027 & 33 & 262 & 0.060 & 0.043 & 29 & 208 & 0.048 & 0.034 & 29 & 122 & 0.034 & 0.020 & 42 & 198 & 0.039 & 0.032 & 17 \\
\hline b12(6) & $4 \times 5$ & 9 & 688 & 0.079 & 0.067 & 15 & 896 & 0.219 & 0.088 & 60 & 424 & 0.064 & 0.041 & 35 & 504 & 0.082 & 0.049 & 40 & 544 & 0.097 & 0.053 & 45 & 904 & 0.126 & 0.088 & 30 \\
\hline $\operatorname{clpl}(3)$ & $6 \times 3$ & 11 & 1218 & 0.040 & 0.033 & 17 & 1579 & 0.043 & 0.043 & 0 & 783 & 0.027 & 0.021 & 22 & 946 & 0.033 & 0.026 & 22 & 618 & 0.017 & 0.017 & 0 & 1584 & 0.043 & 0.043 & 0 \\
\hline jbp(44) & $2 \times 7$ & 11 & 2112 & 0.079 & 0.074 & 7 & 3328 & 0.116 & 0.116 & 0 & 2048 & 0.071 & 0.071 & 0 & 2048 & 0.077 & 0.071 & 7 & 3456 & 0.121 & 0.121 & 0 & 3648 & 0.127 & 0.127 & 0 \\
\hline $\operatorname{luc}(13)$ & $6 \times 4$ & 6 & 86 & 0.058 & 0.056 & 4 & 115 & 0.078 & 0.075 & 4 & 82 & 0.058 & 0.053 & 8 & 85 & 0.058 & 0.055 & 4 & 94 & 0.064 & 0.061 & 4 & 105 & 0.071 & 0.068 & 4 \\
\hline $\max 128(9)$ & $6 \times 4$ & 7 & 269 & 0.091 & 0.088 & 4 & 210 & 0.071 & 0.068 & 4 & 216 & 0.073 & 0.070 & 4 & 260 & 0.092 & 0.085 & 8 & 225 & 0.076 & 0.073 & 4 & 234 & 0.079 & 0.076 & 4 \\
\hline prom2(0) & $6 \times 4$ & 8 & 382 & 0.062 & 0.062 & 0 & 550 & 0.090 & 0.090 & 0 & 308 & 0.050 & 0.050 & & 300 & 0.049 & 0.049 & 0 & 470 & 0.076 & 0.076 & & 306 & 0.050 & 0.050 & \\
\hline
\end{tabular}

TABLE IV

OVERALL RESULTS OF THE SIMULATIONS

\begin{tabular}{|c|c|c|c|c|c|c|c|c|c|c|c|c|c|c|c|c|c|c|c|c|c|c|c|}
\hline Synthesis & Average & Average & $S_{0}$ & $S^{0}$ & $R^{0}$ & $S 1$ & $S^{1}$ & $R^{1}$ & $S R$ & $S R$ & $R^{R}$ & $S_{L}^{L}$ & $S^{L}$ & $R^{L}$ & $S T$ & $S_{T}$ & $R^{T}$ & $G B$ & $G B$ & $R^{B}$ & \multicolumn{3}{|c|}{ Average values } \\
\hline Method & area & $n$ & $S_{C}^{0}$ & $S_{L}^{U}$ & $\% \frac{n}{r \times s}$ & $S_{C}^{1}$ & & $\% \frac{n}{r \times s}$ & $S_{C}^{K}$ & $S_{L}^{R}$ & $\% \frac{n}{r \times s}$ & $S_{C}^{L}$ & & $\% \frac{n}{r \times s}$ & $S_{C}^{T}$ & $S_{L}^{T}$ & $\% \frac{n}{r \times s}$ & $S_{C}^{B}$ & $S_{L}^{B}$ & $\% \frac{n}{r \times s}$ & $S_{C}$ & $S_{L}$ & $\% \frac{R}{r \times s}$ \\
\hline [6] & 63 & 6 & 0.13 & 0.11 & $39 \%$ & 0.08 & 0.04 & $56 \%$ & 0.02 & 0.01 & $35 \%$ & 0.02 & 0.01 & $35 \%$ & 0.05 & 0.03 & $43 \%$ & 0.03 & 0.02 & $45 \%$ & 0.06 & 0.04 & $42 \%$ \\
\hline [17] & 15 & 7 & 0.1 & 0.08 & $20 \%$ & 0.09 & 0.08 & $25 \%$ & 0.06 & 0.06 & $17 \%$ & 0.06 & 0.06 & $17 \%$ & 0.07 & 0.07 & $19 \%$ & 0.07 & 0.07 & $19 \%$ & 0.08 & 0.07 & $20 \%$ \\
\hline
\end{tabular}

The objective here is to identify at the writing time, if common literals and other multiple-choice literals of the function can be mapped on highly critical cells, or not, so that the output is not compromised.

Mitigation for Stuck at Faults: In order to mitigate the sensitivity of a lattice to SAF, we propose the following possible strategy applied to the synthesis method proposed in [6] which has been proven a less sensitive to SAF impact on the output functions: (1) For a given mapped function, if a potential SA0, SA1 faults affects a robust cell identified by the defect injection campaign, the lattice still computes the correct output, thus we do not need any more mitigation with defect tolerant design. (2) However, if an injected defect occurs in a multiple-choice cell, if a different literal can be chosen to increase the robustness, we change the literal with the new one. (3) Otherwise, if the injected SA0 fault is proven as being critical for the output value, the column that contains that defective cell has to be replaced by a spare one. In case of an SA1, the row that contains the defective cell has to be replaced by a spare row. Note that in this case, the output still provides a correct function $f$ from top to bottom, but the function from left to right could be changed and become a function which will not be dual of $f$ anymore.

As an example, consider the lattice synthesized in Figure 7(a) with $f=x_{4} \bar{x}_{5} x_{7}+\bar{x}_{4} x_{6} \bar{x}_{7}+\bar{x}_{4} x_{5} \bar{x}_{6} x_{7}+x_{4} \bar{x}_{6} \bar{x}_{7}+$ $x_{4} x_{6} x_{7}$ by using synthesis method presented in [6]. The example shows one case of mitigation of 3 independent SAD affecting the lattice implementing the function, yielding an approximate $10 \%$ defects. In Figure 7 , critical SA1 cells are marked in blue and SA0 cells a remarked in red.

To avoid output errors due to these SAF we have used the following strategy:

1) Identify robust cells for a given function mapping. Example: the defect in the first row, fifth column is nonsignificant on the value of the output (robust cell). The sensitivity map obtained through defect injection campaign, shows that this cell $\left(x_{4}\right)$ is not sensitive to SA1 for the mapped function.

2) Identify the swapping of literals during synthesis process on a column of a high sensitive cell. Example: the defect in fifth row, fourth column (i.e., blue cell) is sensitive to SA1. We can note that the yellow cell (the fourth row, fourth column cell) contains the literals $\overline{x_{7}}$. By Figure 7(a) we know that we can choose $x_{4}$ instead of $\overline{x_{7}}$. With this new literal $\left(x_{4}\right)$, we have an equivalent lattice where if the blue cell is affected by a SA1 at fabrication time, this will not affect the output of the function.

3) Identify the critical cell for the output value and add a spare column per critical cell. Example: the defect in the third row and second column will influence the value of the output and no swapping operands is possible. Thus the only solution remains to add a spare column (in green) identical to the column containing the SA0 defect, and perform the spare and replace strategy. By using spare columns, the mapping algorithm can eliminate columns of the lattice, with critical cells, susceptible to affect the output value of the function, in case SA defect appear at fabrication or in the field.

Mitigation for Adjacent Cellular Faults: In the case of adjacent cellular fault model, we can note that adjacent cells containing the same controlling literal are robust to faults. For example, consider the lattice depicted in Figure 6(a), and the two variables $x_{4}$ at the end of the first row. The lattice is obviously robust to a L-ACF in the last $x_{4}$ (and to a R-ACF in the forth cell, containing $x_{4}$ ). For this reason, in sensitivity analysis to ACF model, it is of huge importance to maximize the number of adjacent cells with the same controlling literal.

Recall that the properties of the lattice synthesized with [6] guarantee that row and column permutations for a given lattice do not change the Boolean function computed by the switching lattice. Because of the good level of flexibility, we consider the lattice obtained by this synthesis method as a starting point for increasing the number of robust cells without changing the function represented. 
Given Logic Function

$f=x_{4} \overline{x_{5}} x_{7}+\overline{x_{4}} x_{6} \overline{x_{7}}+\overline{x_{4}} x_{5} \overline{x_{6}} x_{7}+x_{4} \overline{x_{6}} \overline{x_{7}}+x_{4} x_{6} x_{7}$

\begin{tabular}{|c|c|c|c|c|}
\hline$x_{4}$ & $\overline{x_{7}}$ & $x_{5}$ & $x_{4}, \overline{x_{7}}$ & $x_{4}$ \\
\hline$\overline{x_{5}}$ & $\frac{x_{6}, \overline{x_{4}}}{\overline{x_{7}}}$ & $\overline{x_{4}}$ & $\overline{x_{7}}$ & $x_{6}$ \\
\hline$x_{7}$ & $\overline{x_{4}}$ & $\begin{array}{c}x_{7}, x_{4}, \\
\overline{x_{6}}\end{array}$ & $\overline{x_{6}}$ & $x_{7}$ \\
\hline$x_{4}$ & $\overline{x_{7}}$ & $\overline{x_{6}}$ & $\frac{x_{4}, x_{6}}{\overline{x_{7}}}$ & $x_{4}$ \\
\hline$x_{4}, x_{7}$ & $x_{6}$ & $x_{7}$ & $x_{4}$ & $\begin{array}{c}x_{4}, x_{6} \\
x_{7}\end{array}$ \\
\hline
\end{tabular}

a)

\begin{tabular}{|l|l|l|l|l|}
\hline$x_{4}$ & $\overline{x_{7}}$ & $x_{5}$ & $x_{4}$ & $x_{4}$ \\
\hline$\overline{x_{5}}$ & $\overline{x_{7}}$ & $\overline{x_{4}}$ & $\overline{x_{7}}$ & $x_{6}$ \\
\hline$x_{7}$ & $\overline{x_{4}}$ & $x_{7}$ & $\overline{x_{6}}$ & $x_{7}$ \\
\hline$x_{4}$ & $\overline{x_{7}}$ & $\overline{x_{6}}$ & $\overline{x_{7}}$ & $x_{4}$ \\
\hline$x_{4}$ & $x_{6}$ & $x_{7}$ & $x_{4}$ & $x_{7}$ \\
\hline
\end{tabular}

b)

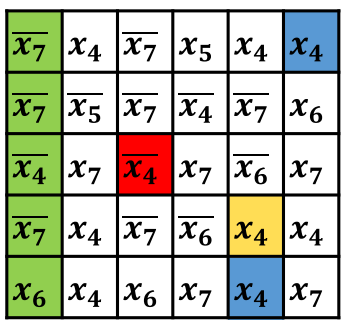

c)
Fig. 7. a) defect-free lattice; b) lattice with defects: SA0 in red and SA1 in blue; and c) lattice with the defect fixed.

Thus, given a lattice synthesized with [6], a possible strategy is to perform column and row permutations in order to maximize the number of adjacent cells containing the same literal. For example, consider the R-ACF, the number of cells with the same literal in the right-adjacent cell in the lattice in Figure 6(a) is 1. Suppose to simply swap the second and the third column, the number of cells with the same literal in the right-adjacent cell becomes 4 (i.e., the number of robust cells is incremented by 3 ). Note also that while the number of robust cells to R-ACF and L-ACF can be increased by column permutations, the number of robust cells to T-ACF and B-ACF can be increased by row permutations. Finally, observe that this method increases the number of robust cells without increasing the dimension of the lattice.

\section{Transient Fault Tolerance}

Before moving on to transient faults, one distinction should be emphasized such that contrary to defects, transient faults are not known in advance and occur in-field. For this reason, transient fault tolerance works in predictive manner. In addition, since nano-crossbars are in the early stage of development, there is data deficiency regarding the occurrence rates and characteristics of transient faults.

First, we follow the same naming conventions of defects (permanent faults) for transient faults in modeling. Accordingly, the effects of transient faults can be categorized with 4 different scenarios:

1) Stuck-at-zero on unused crosspoint produces no effect

2) Stuck-at-zero on used crosspoint removes corresponding input

3) Stuck-at-one on unused crosspoint adds corresponding input

4) Stuck-at-one on used crosspoint produces no effect.

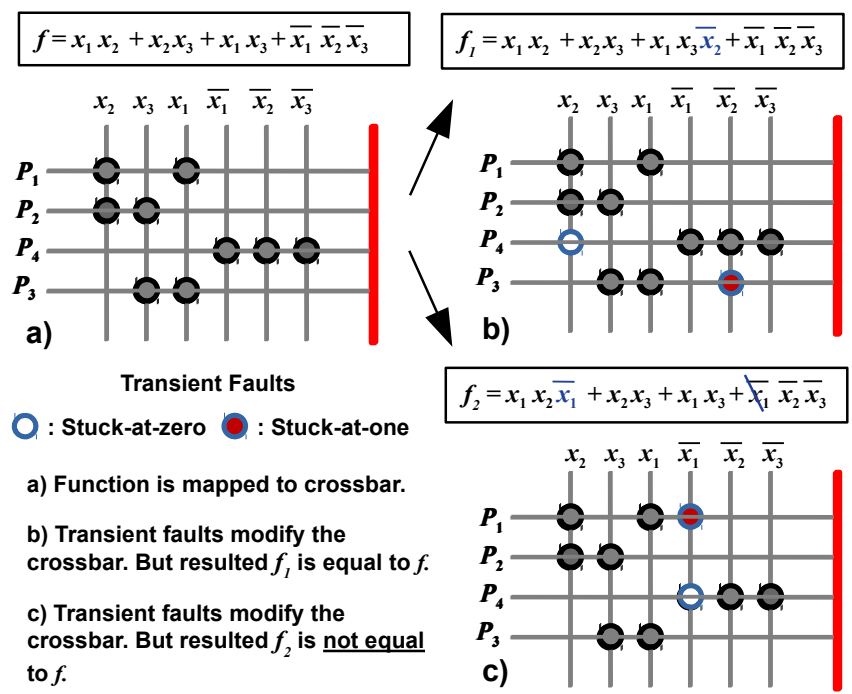

Fig. 8. Transient faults and how inherent tolerance works.

To mitigate faults, first approach is to introduce extra circuit elements to compensate erroneous results. Inserting redundant components can be constructed with adding extra rows and/or columns as shown in [29] [7]. A detailed study of redundancy techniques can be found in [41].

The second approach requires the knowledge of prevalent fault types beforehand so that it is possible to modify the target logic function accordingly. If stuck-at-zero faults are more common, then using the logic function form with least number of inputs implies many unused crosspoints and high tolerance (scenario 1). If stuck-at-one faults are more common, then using the logic function form with the most number of inputs (e.g. using the minterm form) implies many used crosspoints and high tolerance (scenario 4). Nevertheless, trade-off between area size and transient fault tolerance is open to further research.

Finally, logic functions possess an inherent tolerance independent of methodology and benefits from the equivalence of different logic functions. This is an intrinsic feature of the given logic function having many forms. Figure 8 shows how different faults modify the crossbar and resulted logic function is equivalent to the original in Figure 8(b) and not in Figure 8(c). These tolerated faults are limited to certain switches of crossbar and can be found with using algebraic calculations as show in [40]. As for the example in Figure 8 $f=x_{1} x_{2}+x_{2} x_{3}+x_{1} x_{3}+\overline{x_{1}} \overline{x_{2}} \overline{x_{3}}$, every following function is equivalent to the original one:

- $f_{1}=x_{1} x_{2}+x_{2} x_{3} \overline{x_{1}}+x_{1} x_{3}+\overline{x_{1}} \overline{x_{2}} \overline{x_{3}}$

- $f_{2}=x_{1} x_{2} \overline{x_{3}}+x_{2} x_{3}+x_{1} x_{3}+\overline{x_{1}} \overline{x_{2}} \overline{x_{3}}$

- $f 3=x_{1} x_{2}+x_{2} x_{3}+x_{1} x_{3} \overline{x_{2}}+\overline{x_{1}} \overline{x_{2}} \overline{x_{3}}$

For more detailed explanations of determining tolerable switches, calculating the fault tolerance and effects of multioutput functions reader can refer to [40].

Eventually, a more resource hungry approach would be fault sensitivity analysis by performing extensive Monte Carlo simulation and determining every possible case of fault occurrence, yet it is rather costly. As a final word, transient fault tolerance, at the moment, is at its infancy and additional research is needed for best practices. 


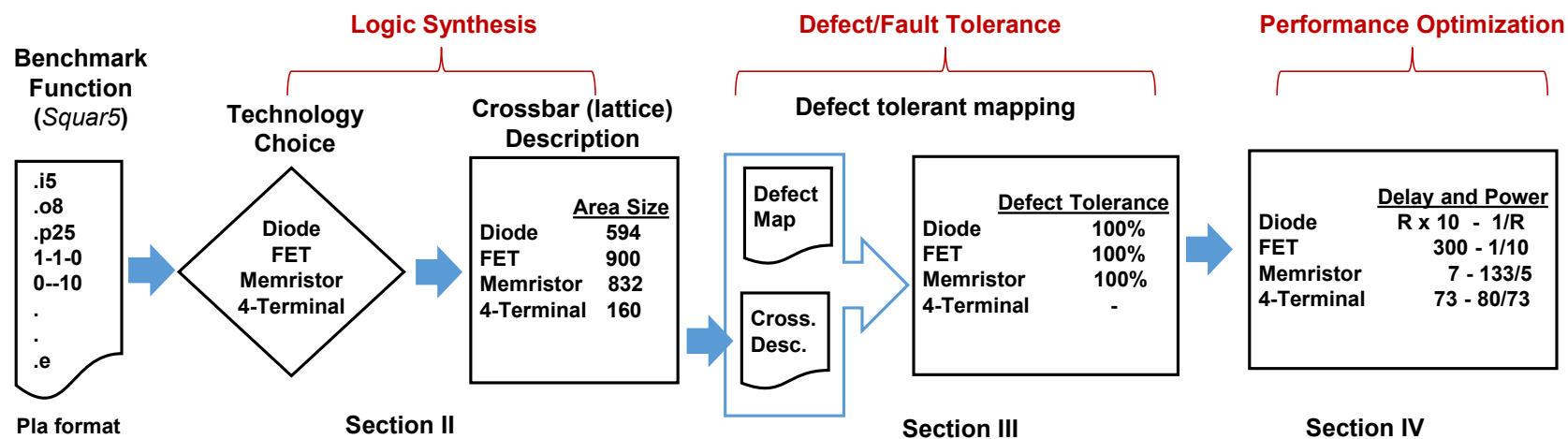

Fig. 9. Whole integrated synthesis pipeline of benchmark function squar5. R denotes the load_resistor.

\section{PERFormance Optimization}

We focus on area-oriented optimization for our methodology. As stated in Section I, main motivation to use nanocrossbars is that they are dense. Thus, logic functions can be realized with smaller area. We first find the minimum sized array, and perform defect tolerance analysis. If it is needed, we increase the area size based on the defect tolerance results. Later, we perform delay and power performance analysis with minor tuning. However, as a downside we loose the benefit of further delay and power minimization [13] [5].

Section III investigates the area performance. Here, we will analyze the delay and power depending on number of products and/or literals. Main purpose is to construct a fair metric for performance comparison. In our previous study [43], we have conducted this analysis for memristive crossbars. All supply voltages of technologies are assumed to be the same. Therefore, comparing their performance behaviors (for a given function) is considered to be sufficient method for integrated synthesis methodology.

1) Delay Analysis: We have used an approach of multiplying resistive and capacitive loads ( $R C$-delay) for delay. This helps us to calculate maximum frequency with 1 /delay. In the delay analysis for diode, we see that "number of columns" and "load resistor" dominate the capacitive load and resistive load respectively. For memristive crossbars, it is proportional to constant $7 \times t_{\text {cycle }}$ (independent from the target function; i.e. latency). Considering FET, we need to calculate resistive loads and capacitive loads from the longest path for the worst-case scenario. Lastly, four-terminal's delay is directly proportional to the longest path on the lattice, same as FET. The longest path on the lattice can be calculated as explained in [6]:

$L_{\text {long_path }}= \begin{cases}R & R \leq 2 \cup C \leq 1 \\ 3 \frac{R-2}{2} \frac{C}{2}+\frac{2+(-1)^{R}+(-1)^{C}}{2} & R>2 \bigcap C>1\end{cases}$

where $R$ : number of rows, $C$ : number of columns, $L_{\text {long_path }}$ : length of the longest path on the lattice.

Finally, Delay performances are proportional to:

- Diode: $\propto($ load_resistor $) \times(\#$ of literals in $f)$

- Memristor: $\propto 7 \times t_{\text {cycle }}$ (latency) [43]

- FET: $\propto$ (degree of the largest product in $f$ and $\left.f^{D}\right)$ $\times\left((\#\right.$ of products in $f)+\left(\#\right.$ of products in $\left.f^{D}\right)+$ \# of literals in $f$ )

- Four-terminal: $\propto L_{\text {long_path }}$
2) Power Analysis: Power consumption is the total energy used in unit time. Therefore we estimate consumed total energy in a period, afterwards we divided it with delay (delay is proportional with a period at maximum frequency). Because we assumed that circuit will be used in maximum frequency. For delay estimation, we will going to use the formulation in above.

The power of diode based crossbar is dominated by static power. So, it is inversely proportional only with a load resistor. Load resistor can be considered as 10 times of diode's inner resistance. Total energy consumption of memristor is proportional to total count, because it is assumed that all memristors are changing their states one time only [43]. Note that, in this memristor based crossbar, sneak path current is minimized [20], so the power consumption caused by it can be neglected. Total energy consumption of FET crossbars is directly proportional to the capacitance of the output node and nodes related to the output node. For four-terminal, we assume all crosspoints consume energy at the worst-case scenario. Finally, we divide energy consumption by the delay to find the complexity of power consumption.

However, effect of the estimated switching activity of an input to the power consumption is not the same for all technologies. For memristor, even though change in inputs does not cause any change for the state of the output, there will be dynamic power consumption. Because every cycle (stage) will be recalculated. Also, since the power of diode is dominated by static power, we didn't include the effect of switching activity to the calculation of power consumption. For only FET and four-terminal, we include the estimation of switching activity by multiplying it with 0.5 . (Notice that, supply voltages are assumed as the same.)

Finally, Power performances are proportional to:

- Diode: $\propto 1$ / (load_resistor)

- Memristor: $\propto$ (total memristor count in crossbar $) / 7$ [43] [39]

- FET: $\propto 0.5 /\left(\right.$ degree of the largest product in $f$ and $\left.f^{D}\right)$ (@ maximum frequency)

- Four-terminal: $\propto(0.5$ (\# of products in rows $) \times$ (\# of products in columns)) / $L_{\text {long_path }}$

\section{A Case Study for Benchmark Function Squar5}

In this section, we demonstrate the whole process with an example. We have chosen the benchmark function Squar5 
which has 5 inputs, 25 products and 8 outputs in PLA format. Diagram of the process is given in Figure 9.

First, we need to evaluate different technologies such as diode, FET, memristor or four-terminal. Using logic synthesis, we can generate function descriptions in crossbar (lattice) form and obtain area sizes utilizing equations in Section III. Results are as follows:

Diode: (\# of products of all $\left.\left.f_{\text {oi }}\right)+n\right) \times$ $((\#$ of literals in $f)+n)=(25+8) \times(10+8)=$ 594.

Memristor: ((\# of products of all $\left.\left.f_{o i}\right)+n\right) \times$ $((\#$ of literals in $f)+2 n)=(25+8) \times(10+16)=858$. However, our proposed greedy algorithm PGA decreases the size to 832 .

FET: (\# of literals in $f+n) \times$ $\left(\left(\#\right.\right.$ of products of all $\left.f_{\text {oi }}\right)+\left(\#\right.$ of products of all $\left.\left.f_{\text {oi }}^{D}\right)\right)$ $=(10+8) \times(25+25)=900$. Coincidentally, its dual also has the same number of products.

Four-terminal: (degree of the largest product in $\left.f_{o i}^{D}\right) \times$ (\# of products of all $\left.f_{\text {oi }}+n-1\right)=5 \times(25+8-1)=160$. First term is chosen according to the product which has the maximum number of literals.

Secondly, we use function descriptions and defect map of a crossbar. Applying the defect tolerant logic mapping methods in Section IV, it is possible to measure defect/fault performance. Key point is that diode, FET and memristor have a rich literature of methods for reaching $100 \%$ defect tolerance for defect rates up to $10 \%$ [41] [43]; nevertheless four-terminal is at its infancy in terms of defect tolerance. In this paper, defect performance of only single output functions are studied and we are planning to extend the work into multi output functions as well in the future.

Lastly, we conduct a performance optimization specific to technology dependent delay and power parameters of crossbars. Since related equations are presented in immediate section, only result are given:

Diode: Delay is $R \times 10$ (Load_resistor is shown with $R$ ). Power is $1 / R$. If assume $R$ is 10 times than a diode's inner resistance, then Delay is 100 and Power is $1 / 10$.

Memristor: Delay (latency) is constant (independent from

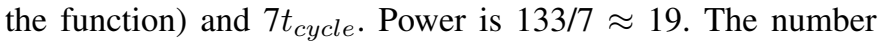
133 denotes the number of memristors used in the crossbar.

FET: Delay is 300 and power is $1 / 10$. Including the next four-terminal, FET has the largest delay.

Four-terminal: The longest path is 73 , so is the delay. Power is $80 / 73 \approx 1$.

Note that, values only lights the complexity of delay and power, they are not real elapsed time and power consumption. Here we assume, they all fabricated with same technology. For instance, in order to calculate the delay of diode crossbars, we need to know value of $R$ and the source voltage.

To provide an overall evaluation, four-terminal is the most advantageous choice in terms of area size. However, defect tolerance is poor especially regarding the complexity and computation power needed to conduct experiments for fourterminal. Delay of memristive crossbars is predictable, it doesn't depend on function. Therefore, it could be considered as the most advantageous choice in terms of delay. For power consumption, diode seems to have the least value, yet it is static power also depends source voltage. On the other hand, FET crossbars has only dynamic power consumption with complementary architecture.

\section{CONCLUSION AND DISCUSSION}

In conclusion, we have presented an integrated synthesis methodology regarding every design steps of a crossbar circuit including novel optimization and defect tolerance algorithms for each step. Since the main motivation of crossbar technologies is that they are able produce denser circuit structures comparing to the conventional technologies, the synthesis methodology takes area optimization as base. In addition to that, defect tolerance analysis gets the size of the crossbar as the input. However, devising area-oriented optimization causes only having course delay and power analysis/optimization. An extension of the selection of delay- or power-oriented optimizations will increase the performance optimization capability of the methodology.

In logic synthesis section, comparison of four crossbar types with multi-output logic functions is firstly presented. Also, a new technique of logic synthesis for memristor based crossbars is presented. Since developing new efficient algorithms for logic synthesis is in progress and also the area sizes are depending on different parameters of a logic, there has not been a verdict on this race.

In defect tolerance section, we focus on four-terminal architectures and design a novel sensitivity analysis and fault tolerance approach. First step of the method locates the problematic cells and guides the second step through introducing preventive measures such as reassignment of cell (if possible) or adding redundant columns/rows to the lattice. Currently, method is limited in term of input size, so an important improvement to sensitivity analysis would be to increase the number of variables the method can manage.

In performance optimization section, effects of the crossbar size and function parameters to the delay and power are presented. Even though room for optimizations of delay and power are limited due to the area-oriented optimization, the paper presents a clear projections to the designer. However the real values in the crossbar technologies (for different types) is in process of evolving. Certain studies are in proof of concept level. For instance, pitch sizes were given as micrometers in the early 2000s [18] [34], yet they are reduced to tens of manometers [20]. Also, a recent study [13] realizes fourterminal switch based crossbar (lattice) with $65 \mathrm{~nm}$ CMOSprocess for a proof of concept. As expected, the situation is also same for the comparison on the delay and power analysis. This is the inherent reason that this study is independent from technology nodes, thus it can be implemented to the every technology. 3D printing electronics could be a promising candidate for comparing the crossbar types with actual values.

As a future work, a fully automated software tool for the synthesis methodology is planned to be developed. It will take the target function, the used technology, and the performance specifications (area, delay and/or power consumption) as input and return the optimized crossbar-arrays structure as an output. This software tool, to be developed, will benefit from the 
optimization algorithms introduced in this study. It will also produce the mapping using technology specification data. The tool is expected to be updated based on established experimental results and novel algorithms. Another direction could be the study of transient faults on lattices, so that it can generate complete defect tolerance analysis. The future study should evaluate how the redundancies in lattices can limit the area overhead.

Acknowledgement: We thank Dr. Levent Aksoy for his contribution to synthesis process of multi-output functions with four-terminal based switching arrays.

\section{REFERENCES}

[1] L. Aksoy and M. Altun, "Novel methods for efficient realization of logic functions using switching lattices," IEEE Transactions on Computers, vol. 69, no. 3, pp. 427-440, 2019.

[2] L. Aksoy and M. Altun, "A satisfiability-based approximate algorithm for logic synthesis using switching lattices," in Design, Automation \& Test in Europe Conference \& Exhibition (DATE). IEEE, 2019, pp. $1637-1642$.

[3] L. Aksoy and M. Altun, "A novel method for the realization of complex logic functions using switching lattices," in Design, Automation \& Test in Europe Conference \& Exhibition (DATE). IEEE, 2020.

[4] D. Alexandrescu, M. Altun, L. Anghel, A. Bernasconi, V. Ciriani, L. Frontini, and M. Tahoori, "Synthesis and performance optimization of a switching nano-crossbar computer," in Euromicro Conference on Digital System Design (DSD). IEEE, 2016, pp. 334-341.

[5] M. Altun, I. Cevik, A. Erten, O. Eksik, M. Stan, and C. A. Moritz, "Nano-crossbar based computing: Lessons learned and future directions," in Design, Automation \& Test in Europe Conference \& Exhibition (DATE). IEEE, 2020.

[6] M. Altun and M. D. Riedel, "Logic synthesis for switching lattices," IEEE Transactions on Computers, vol. 61, no. 11, pp. 1588-1600, 2012.

[7] S. Baranov, I. Levin, O. Keren, and M. Karpovsky, "Designing fault tolerant FSM by nano-PLA," in 15th IEEE International On-Line Testing Symposium (IOLTS). IEEE, 2009, pp. 229-234.

[8] R. Ben-Hur, R. Ronen, A. Haj-Ali, D. Bhattacharjee, A. Eliahu, N. Peled, and S. Kvatinsky, "SIMPLER MAGIC: synthesis and mapping of inmemory logic executed in a single row to improve throughput," IEEE Transactions on Computer-Aided Design of Integrated Circuits and Systems, 2019.

[9] A. Bernasconi, "Composition of switching lattices and autosymmetric boolean function synthesis," in 2016 Euromicro Conference on Digital System Design (DSD). IEEE, 2017.

[10] A. Bernasconi, V. Ciriani, L. Frontini, V. Liberali, G. Trucco, and T. Villa, "Logic synthesis for switching lattices by decomposition with p-circuits," in 2016 Euromicro Conference on Digital System Design (DSD). IEEE, 2016, pp. 423-430.

[11] A. Bernasconi, V. Ciriani, L. Frontini, and G. Trucco, "Synthesis on switching lattices of dimension-reducible boolean functions," in 2016 IFIP/IEEE International Conference on Very Large Scale Integration (VLSI-SoC). IEEE, 2016, pp. 1-6.

[12] J. Borghetti, Z. Li, J. Straznicky, X. Li, D. A. Ohlberg, W. Wu, D. R. Stewart, and R. S. Williams, "A hybrid nanomemristor/transistor logic circuit capable of self-programming," Proceedings of the National Academy of Sciences, vol. 106, no. 6, pp. 1699-1703, 2009.
[13] I. Cevik, L. Aksoy, and M. Altun, "Cmos implementation of switching lattices," in Design, Automation \& Test in Europe Conference \& Exhibition (DATE). IEEE, 2020, pp. 274-277.

[14] C.-Y. Chen, H.-C. Shih, C.-W. Wu, C.-H. Lin, P.-F. Chiu, S.-S. Sheu, and F. T. Chen, "RRAM defect modeling and failure analysis based on march test and a novel squeeze-search scheme," IEEE Transactions on Computers, vol. 64, no. 1, pp. 180-190, 2015.

[15] Y. Chen, G.-Y. Jung, D. A. Ohlberg, X. Li, D. R. Stewart, J. O. Jeppesen, K. A. Nielsen, J. F. Stoddart, and R. S. Williams, "Nanoscale molecularswitch crossbar circuits," Nanotechnology, vol. 14, pp. 462-468, 2003.

[16] A. D. Friedman, "Easily testable iterative systems," IEEE Transactions on Computers, vol. 100, no. 12, pp. 1061-1064, 1973.

[17] G. Gange, H. Søndergaard, and P. J. Stuckey, "Synthesizing optimal switching lattices," ACM Transactions on Design Automation of Electronic Systems (TODAES), vol. 20, no. 1, pp. 1-14, 2014.

[18] Y. Huang, X. Duan, Y. Cui, L. J. Lauhon, K.-H. Kim, and C. M. Lieber, "Logic gates and computation from assembled nanowire building blocks," Science, vol. 294, no. 5545, pp. 1313-1317, 2001.

[19] W. Kang, W. Zhao, Z. Wang, Y. Zhang, J.-O. Klein, Y. Zhang, C. Chappert, and D. Ravelosona, "A low-cost built-in error correction circuit design for STT-MRAM reliability improvement," Microelectronics Reliability, vol. 53, no. 9-11, pp. 1224-1229, 2013.

[20] K.-H. Kim, S. Gaba, D. Wheeler, J. M. Cruz-Albrecht, T. Hussain, N. Srinivasa, and W. Lu, "A functional hybrid memristor crossbararray/CMOS system for data storage and neuromorphic applications," Nano letters, vol. 12, no. 1, pp. 389-395, 2012.

[21] S. Kvatinsky, D. Belousov, S. Liman, G. Satat, N. Wald, E. G. Friedman, A. Kolodny, and U. C. Weiser, "MAGIC-memristor-aided logic," IEEE Transactions on Circuits and Systems II: Express Briefs, vol. 61, no. 11, pp. 895-899, 2014

[22] C. Li, D. Belkin, Y. Li, P. Yan, M. Hu, N. Ge, H. Jiang, E. Montgomery, P. Lin, Z. Wang et al., "Efficient and self-adaptive in-situ learning in multilayer memristor neural networks," Nature communications, vol. 9, no. 1 , pp. $1-8,2018$

[23] P. C. McGeer, J. V. Sanghavi, R. K. Brayton, and A. SangiovanniVicentelli, "ESPRESSO-SIGNATURE: A new exact minimizer for logic functions," IEEE Transactions on Very Large Scale Integration (VLSI) Systems, vol. 1, no. 4, pp. 432-440, 1993.

[24] M. C. Morgul, O. Tunali, M. Altun, L. Frontini, V. Ciriani, E. I. Vatajelu, L. Anghel, C. A. Moritz, M. R. Stan, and D. Alexandrescu, "Integrated synthesis methodology for crossbar arrays," in 14th IEEE/ACM International Symposium on Nanoscale Architectures (NANOARCH), 2018, pp. 91-97.

[25] M. C. Morgül and M. Altun, "Anahtarlamalı nano dizinler ile lojik devre tasarımı ve boyut optimizasyonu logic circuit design with switching nano arrays and area optimization," in ELECO, 2014.

[26] M. C. Morgul and M. Altun, "Synthesis and optimization of switching nanoarrays," in 2015 IEEE 18th International Symposium on Design and Diagnostics of Electronic Circuits \& Systems (DDECS). IEEE, 2015, pp. 161-164.

[27] M. Nicolaidis, L. Anghel, and N. Achouri, "Memory defect tolerance architectures for nanotechnologies," Journal of Electronic Testing, vol. 21, no. 4, pp. 445-455, 2005.

[28] F. Peker and M. Altun, "A fast hill climbing algorithm for defect and variation tolerant logic mapping of nano-crossbar arrays," IEEE Transactions on Multi-Scale Computing Systems, vol. 4, no. 4, pp. 522532, 2018.

[29] W. Rao, A. Orailoglu, and R. Karri, "Logic level fault tolerance approaches targeting nanoelectronics PLAs," in 2007 Design, Automation \& Test in Europe Conference \& Exhibition. IEEE, 2007, pp. 1-5.

[30] S. Safaltin, O. Gencer, M. C. Morgul, L. Aksoy, S. Gurmen, C. A. Moritz, and M. Altun, "Realization of four-terminal switching lattices: Technology development and circuit modeling," in 2019 Design, Automation \& Test in Europe Conference \& Exhibition (DATE). IEEE, 2019, pp. 504-509.

[31] S. Shirinzadeh, M. Soeken, P.-E. Gaillardon, and R. Drechsler, "Logic synthesis for RRAM-based in-memory computing," IEEE Transactions on Computer-Aided Design of Integrated Circuits and Systems, vol. 37, no. 7, pp. 1422-1435, 2017.

[32] A. M. S. Shrestha, S. Tayu, and S. Ueno, "Orthogonal ray graphs and nano-PLA design," in 2009 IEEE International Symposium on Circuits and Systems (ISCAS). IEEE, 2009, pp. 2930-2933.

[33] G. Snider, "Computing with hysteretic resistor crossbars," Applied Physics A: Materials Science \& Processing, vol. 80, no. 6, pp. 11651172, 2005.

[34] G. Snider, P. Kuekes, T. Hogg, and R. S. Williams, "Nanoelectronic architectures," Applied Physics A, vol. 80, no. 6, pp. 1183-1195, 2005. 
[35] G. Snider, P. Kuekes, and R. S. Williams, "CMOS-like logic in defective, nanoscale crossbars," Nanotechnology, vol. 15, no. 8, p. 881, 2004.

[36] D. B. Strukov and K. K. Likharev, "CMOL FPGA: a reconfigurable architecture for hybrid digital circuits with two-terminal nanodevices," Nanotechnology, vol. 16, no. 6, p. 888, 2005.

[37] M. B. Tahoori, "A mapping algorithm for defect-tolerance of reconfigurable nano-architectures," in IEEE/ACM International Conference on Computer-Aided Design (ICCAD). IEEE, 2005, pp. 668-672.

[38] P. L. Thangkhiew, A. Zulehner, R. Wille, K. Datta, and I. Sengupta, "An efficient memristor crossbar architecture for mapping boolean functions using binary decision diagrams (bdd)," Integration, vol. 71, pp. 125-133, 2020.

[39] M. Traiola, M. Barbareschi, and A. Bosio, "Estimating dynamic power consumption for memristor-based CiM architecture," Microelectronics Reliability, vol. 80, pp. 241-248, 2018.

[40] O. Tunali and M. Altun, "Permanent and transient fault tolerance for reconfigurable nano-crossbar arrays," IEEE Transactions on ComputerAided Design of Integrated Circuits and Systems, vol. 36, no. 5, pp. 747-760, 2016.

[41] O. Tunali and M. Altun, "A survey of fault-tolerance algorithms for reconfigurable nano-crossbar arrays," ACM Computing Surveys (CSUR), vol. 50 , no. 6 , pp. $1-35,2017$.

[42] O. Tunali and M. Altun, "Logic synthesis and defect tolerance for memristive crossbar arrays," in 2018 Design, Automation \& Test in Europe Conference \& Exhibition (DATE). IEEE, 2018, pp. 425-430.

[43] O. Tunali, M. C. Morgul, and M. Altun, "Defect-tolerant logic synthesis for memristor crossbars with performance evaluation," IEEE Micro, vol. 38, no. 5, pp. 22-31, 2018.

[44] L. Xie, H. A. Du Nguyen, M. Taouil, S. Hamdioui, and K. Bertels, "Fast boolean logic mapped on memristor crossbar," in 2015 33rd IEEE International Conference on Computer Design (ICCD). IEEE, 2015, pp. 335-342.

[45] L. Xie, H. A. Du Nguyen, M. Taouil, S. Hamdioui, and K. Bertels, "A mapping methodology of boolean logic circuits on memristor crossbar," IEEE Transactions on Computer-Aided Design of Integrated Circuits and Systems, vol. 37, no. 2, pp. 311-323, 2017

[46] H. Yan, H. S. Choe, S. Nam, Y. Hu, S. Das, J. F. Klemic, J. C. Ellenbogen, and C. M. Lieber, "Programmable nanowire circuits for nanoprocessors," Nature, vol. 470, no. 7333, pp. 240-244, 2011.

[47] S. Yang, Logic synthesis and optimization benchmarks user guide version 3.0. Research Triangle Park, NC, USA: Microelectronics Center of North Carolina (MCNC), 1991.

[48] J. Yao, H. Yan, S. Das, J. F. Klemic, J. C. Ellenbogen, and C. M. Lieber, "Nanowire nanocomputer as a finite-state machine," Proceedings of the National Academy of Sciences, vol. 111, no. 7, pp. 2431-2435, 2014.

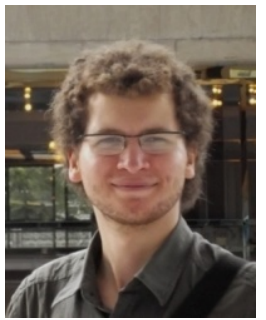

Muhammed Ceylan Morgul received his BSc degree in electronics and comm. eng. in 2014, and MSc degree in electronics eng. in 2017 at Istanbul Technical University. $\mathrm{He}$ is currently a $\mathrm{PhD}$ student in Electrical Engineering at the University of Virginia. He has been principal investigator of one TUBITAK, and researcher of EU-H2020-RISE, SRC-JUMP and TUBITAK projects, in Turkey, USA, France, Portugal and Malaysia. His current research interests include reliability and emerging computing.

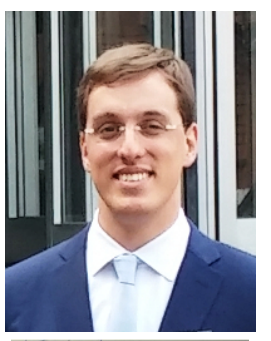

Luca Frontini received the Laurea degree in physics in 2015 (University of Milan), in 2019 he received the Ph.D. in Computer Science (University of Milan). He is currently a postdoc at University of Milan. His main interests are: logic synthesis of emerging technologies, design of mixed-signal circuits, VLSI design, rad-hard electronics, and integrated photonic devices.

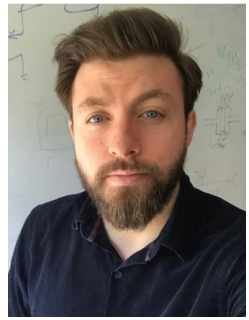

Onur Tunalı received his BSc degree in mathematics at Istanbul University and MSc degree in Nanoscience and Nanoengineering at Istanbul Technical University. He has worked as a researcher in various projects including TUBITAK and EU H2020 RISE. His current research interests include logic synthesis, re-configurable nano-crossbars, algorithm design,emerging computing, and reliability.

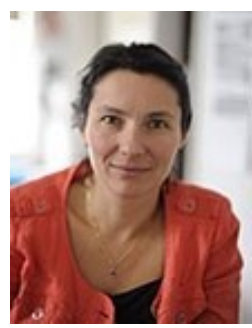

Lorena Anghel is Scientific Director and a Full professor at Grenoble Institute of Technology, and a member of the research staff of SPINTEC Laboratory. She received the $\mathrm{PhD}$ from Grenoble Institute of Technology in 2000, Cum Laudae. Her research interests include hardware design and test of neural networks, on-line testing, fault tolerance, and digital reliable design and verification. She has been involved in European Projects (FP5 and FP7) and French National projects ANR,

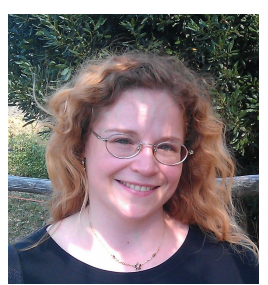

Valentina Ciriani received the Laurea degree (in 1998) and the Ph.D. degree (in 2003) in Compute Science from the University of Pisa. She is currently an Associate Professor in Computer Science with the Department of Computer Science, Università degli Studi di Milano. Her research interests include combinational logic for emerging technologies, VLSI design of low power circuits, and test of Boolean circuits. She has authored more than 90 research papers and books chapters.

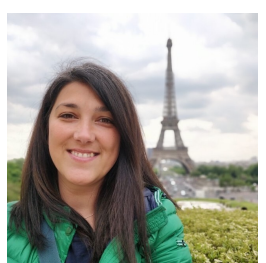

Elena Ioana Vatajelu received her $\mathrm{PhD}$ degree in electronics engineering from Universitat Politecnica de Catalunya, Barcelona in 2011. Is currently a CNRS researcher within TIMA Laboratory in Grenoble, France. Her current research includes emerging memory technologies with special emphasis on their use in architectures for emerging computing paradigms; mainly the characterization of fabrication induced process variability, fault modeling and defect characterization; design for- reliability, design-for-test and design-for-security

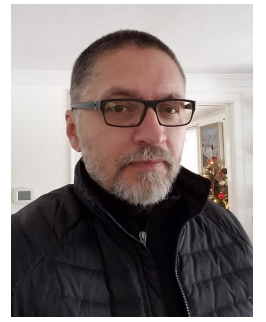

Csaba Andras Moritz is a Professor of Electrical and Computer Engineering at the University of Massachusetts Amherst. He is the founder and chairman of BlueRISC Inc., a leading system-assurance company, and EPRIVO.com in digital privacy. His interests include nano fabrics beyond 2D CMOS and circuit styles with emerging technology, new models of computation and inference for AI and their realization through emerging technology, and AI-driven cyber-security and system assurance.

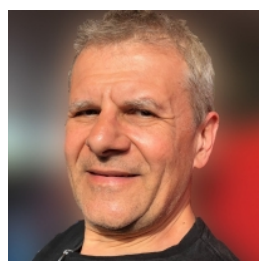

Mircea R. Stan is the Virginia Microelectronics Consortium Professor at the University of Virginia. $\mathrm{He}$ has received the 2018 Influential ISCA Paper Award, the NSF CAREER award in 1997 and was co-author on best paper awards at ASILOMAR19, LASCAS19, SELSE17, ISQED09, GLSVLSI06, ISCA03 and SHAMAN02 and on IEEE Micro Top Picks in 2008 and 2003. He gave keynotes at several conferences, and is Associate Editor-in-Chief for the IEEE TVLSI, Senior Editor for the IEEE TNANO, Associate Editor for IEEE Design\&Test.

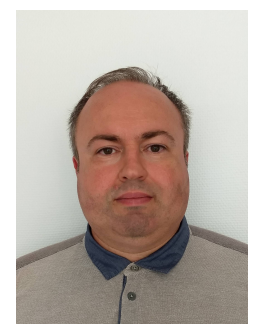

Dan Alexandrescu (IEEE Member'07-Senior Member'13) is the CEO of IROC Technologies. Dan holds a Ph.D in Microelectronics from INPG, Grenoble Institute of Technology, France. He specializes in the design, optimization and improvement of highlyreliable microelectronic circuits. He contributed to the organization of reliability-centric workshops and symposia and he prepared many publications in the field of reliability and radiation-induced effects.

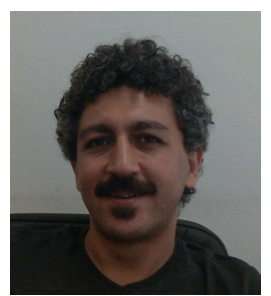

Mustafa Altun He received the Ph.D. degree in electrical engineering with the Ph.D. minor in mathematics from the University of Minnesota in 2012. Since 2013, he has been a faculty member of Istanbul Technical University and runs the Emerging Circuits and Computation (ECC) Group 